\title{
Object-based visual selective attention and perceptual organization
}

\author{
STEPHEN E. WATSON \\ Armstrong Laboratory, Brooks Air Force Base, San Antonio, Texas \\ and \\ ARTHUR F. KRAMER \\ Beckman Institute, University of Illinois, Urbana, Illinois
}

\begin{abstract}
We report the results of four experiments that were conducted to examine both the representations that provide candidate entities available for object-based attentional selection and the influence of bottomup factors (i.e., geometric and surface characteristics of objects) and top-down factors (i.e., context and expectancies) on the selection process. Subjects performed the same task in each of the experiments. They were asked to determine whether two target properties, a bent end and an open end of a wrench, appeared in a brief display of two wrenches. In each experiment, the target properties could occur on a single wrench or one property could occur on each of two wrenches. The question of central interest was whether a same-object effect (faster and/or more accurate performance when the target properties appeared on one vs. two wrenches) would be observed in different experimental conditions. Several interesting results were obtained. First, depending on the geometric (i.e., concave discontinuities on object contours) and surface characteristics (i.e., homogeneous regions of color and texture) of the stimuli, attention was preferentially directed to one of three representational levels, as indicated by the presence or absence of the same-object effect. Second, although geometric and surface characteristics defined the candidate objects available for attentional selection, top-down factors were quite influential in determining which representational level would be selected. Third, the results suggest that uniform connectedness plays an important role in defining the entities available for attention selection. These results are discussed in terms of the manner in which attention selects objects in the visual environment.
\end{abstract}

A number of spatial metaphors, such as spotlights (B. A. Eriksen \& C. W. Eriksen, 1974; Posner, 1980), zoom lenses (C.W. Eriksen \& St. James, 1986; C. W. Eriksen \& Yeh, 1985) and gradients (Downing \& Pinker, 1985; LaBerge \& Brown, 1989), have been used to characterize the manner in which attention is distributed in the visual field. These metaphors suggest that visual attention is unitary in nature, can be flexibly deployed to large and small areas of visual space, and is characterized by a particular shape (i.e., circular or elliptical) that is independent of the objects and structure in the visual environment. Indeed, such metaphors have been quite successful in accounting for a variety of empirical observations, such as spatial cuing effects (Bashinski \& Bacharach, 1980; Posner, 1980), distractor interference effects (B. A.

This research was supported by a cooperative research agreement with the Army Research Laboratory (DAAL01-96-2-0003) and the Air Force Office of Scientific Research through the Learning Abilities Measurement Program and Armstrong Laboratory. The authors thank David Irwin, Jan Theeuwes, and Shaun Vecera for their helpful comments on a previous draft of this manuscript. The authors also thank Richard Walker for programming support. Correspondence should be addressed to S. E. Watson, Armstrong Laboratory, Brooks Air Force Base, San Antonio, TX $78235-5352$ (e-mail: watson@alhem.brooks. af.mil), or to A. F. Kramer, Beckman Institute, University of Illinois, 405 North Mathews Ave., Urbana, IL 61801 (e-mail: akramer@s.psych. uiuc.edu).
Eriksen \& C. W. Eriksen, 1974; Ruthruff \& Miller, 1995), and divided attention among spatial locations (J. E. Hoffman, Houck, McMillian, Simons, \& Oatman, 1985; J. E. Hoffman \& Nelson, 1981; Kramer \& Hahn, 1995).

Space-based models of attention have also received substantial support from neuropsychological and human electrophysiological studies. For example, patients with unilateral visual neglect, a syndrome associated with parietal lobe damage to a single hemisphere, have difficulty responding to stimuli presented on the side of space contralateral to their lesion. Interestingly, failure to respond to contralesional stimuli is not usually accompanied by a loss of vision on the affected side. However, cuing stimuli in the affected field has been found to reduce the unilateral neglect (Posner, Walker, Friedrich, \& Rafal, 1984; Riddoch \& Humphreys, 1983). This finding suggests that neglect might result in a reduced ability to direct attention to the contralesional side of space. Studies of human electrophysiology and, more specifically, event-related brain potentials (ERP) have also been supportive of space-based conceptions of attention. Indeed, several components of the ERP, such as the P100 and N100, appear to be uniquely sensitive to the distribution of attention to particular locations in the visual field (Hillyard et al., 1996; Mangun \& Hillyard, 1990).

Although space-based models have provided a good account of a number of attentional phenomena over the past 
several decades, it has become increasingly clear that attention can also be used to select objects and perceptual groups rather than simply regions of space. Indeed, the research that supports the notion of object-based visual attention can be viewed as descending from the Gestaltists, such as Wertheimer (1923), who demonstrated the importance of principles of grouping in visual and auditory perception, and Neisser (1967), who proposed that the visual field is initially preattentively segregated into figural units or objects on the basis of Gestalt principles and that focused attention is then employed to analyze specific objects in more detail (see also Moore \& Egeth, 1997).

Evidence in support of object-based attentional selection has been obtained in focused attention, divided attention, and spatial cuing paradigms with both normal subjects and human lesion patients. One of the classic studies was reported by Duncan (1984). In his paradigm, subjects were presented with two overlapping objects, a box and a line, and were asked to identify either two properties of one object (i.e., the texture and orientation of the line or the size and side of a gap in the box) or one property of the line and another property of the box. Identification was more accurate when the properties were located on a single object than when one property was located on one object and the other property appeared on the other object. A critical feature of Duncan's (1984) paradigm was that the target properties that subjects were to identify were equally spaced, regardless of whether they were located on one or two objects. Thus, a spacebased model that postulates that attention is focused independently of the objects or structure in the environment (i.e., a spotlight, gradient, or zoom lens) cannot account for this same-object performance effect.

Since Duncan's (1984) groundbreaking study, there have been a number of additional reports of faster or more accurate identification of multiple properties when these properties are located on a single object relative to when they occur on different objects (Kramer, Weber, \& Watson, 1997; Kramer, Wickens, \& Donchin, 1985; Lavie \& Driver, 1996; Vecera \& Farah, 1994; Weber, Kramer, \& Miller, 1997). Furthermore, same-object effects (i.e., performance is faster and/or more accurate when subjects identify two properties on a single object than when they identify one property on each of two different objects) have been obtained even for partially occluded objects (Behrmann, Zemel, \& Mozer, 1998; Davis \& Driver, 1997) and for objects defined by experimenter instructions rather than image-based properties (Baylis, 1994; Baylis \& Driver, 1995; Yantis, 1992).

Evidence for object-based selection has also been obtained in focused attention and spatial cuing paradigms. Kramer and Jacobson (1991; see also Baylis \& Driver, 1992) found that distractor interference effects could be substantially reduced if the distractors and targets were located on different objects. Egly, Driver, and Rafal (1994; see also Egly, Rafal, Driver, \& Starrveveld, 1994; Stuart, Maruff, \& Currie, 1997; Yantis \& Moore, 1995) reported that attention could be reallocated more quickly when a target was located at an uncued location within a cued object than when the target appeared at the same distance from the originally cued location but in an uncued object. The phenomenon of inhibition of return-that is, increased reaction time when a target appears in a location that has recently been attended - also appears to be object based. Tipper, Brehaut, and Driver (1990) reported that inhibition appears to follow a moving object rather than being associated with the original location in which the object was attended (see also Tipper, Driver, \& Weaver, 1991; Weaver, Lupiàñez, \& Watson, 1998).

Evidence in support of object-based visual selection has also been provided in a number of recent neuropsychological studies. For example, several studies have reported cases of neglect not only for the side of the visual field contralateral to the patient's lesion but also for a particular side of an object independent of the area of the visual field in which the object appeared (Behrmann \& Moscovitch, 1994; Driver \& Halligan, 1991; Tipper \& Behrmann, 1996). Other studies have found that extinction effects, reporting only one of two simultaneously presented objects, can be eliminated when the objects are linked (Humphreys, Olson, Romani, \& Riddoch, 1996; Humphreys \& Riddoch, 1993; Rorden, Mattingley, Karnath, \& Driver, 1997).

\section{REPRESENTATIONS AVAILABLE FOR OBJECT-BASED ATTENTIONAL SELECTION}

Although it is now clear that attention can select objects rather than spatial regions per se, there has been relatively little systematic empirical research on the nature of the object representations from which attention can select. Indeed, in the great majority of the studies in which object-based attentional selection has been examined, objects have often been defined rather intuitively or by invoking Gestalt principles of grouping, such as proximity, similarity, good continuation, closure, and so on (Duncan, 1984; Kahneman \& Henik, 1981; Kahneman, Treisman, \& Gibbs, 1992; Kramer \& Jacobson, 1991; Tipper \& Behrmann, 1996; Treisman, Kahneman, \& Burkell, 1983).

However, there have been several notable exceptions. For example, Baylis and Driver (1993) had subjects judge the relative location of two contours. They found that the relative location of two objects disrupted the judgment of the relative location of object parts, but the reverse did not occur. That is, visual attention was constrained by a hierarchical coding of scene-based and object-based representations. Although this proposal, and the empirical data that support it, provide important insights into the judgments of relative positions of objects and object parts, it remains to be determined whether it will apply equally well to judgments of object attributes other than relative position.

Logan (1997) proposed the CODE theory of visual attention, which attempts to integrate space-based and object-based notions of attention. CODE accomplishes 
this integration by clustering nearby stimuli into perceptual groups that are both objects and regions of space. The CODE model has been successfully employed to account for the results obtained in a variety of different spatial attentional paradigms. However, the fact that CODE accomplishes the creation of objects solely via grouping by proximity also limits the situations in which it can be applied. For example, the present instantiation of the CODE model cannot account for the same-object effect (i.e., faster and/or more accurate performance when two equally spaced properties are located on a single object relative to when the properties are located on two different objects; see Baylis \& Driver, 1993; Duncan, 1984; Kramer \& Watson, 1996; Lavie \& Driver, 1996).

Driver and Baylis (1995; see also Baylis \& Driver, 1995) conducted a series of experiments to examine the role of attention in figure-ground segmentation, shape description, and object part decomposition. They obtained several important results relevant to the nature of the representations available for object-based attentional selection. First, subjects were unable to selectively attend to the dividing edge between two regions of an ambiguous figureground display. Instead, they selected the entire figure to which the edge had been assigned. Furthermore, edges were substantially more likely to be assigned to convex than to concave regions of the ambiguous figure-ground display. Second, Driver and Baylis found that the detection of symmetry differences among convex parts of an object appears to take place in parallel - that is, symmetry judgments are not influenced by the number of convex parts of an object. On the other hand, symmetry comparisons of concave parts appear to be slow and serial. Finally, they found that such judgments are much more time consuming and error prone for multiple objects than for a single object, even when the multiple objects are of equivalent size to the single object.

The data obtained by Driver and Baylis (1995) begin to define the nature of the object representations from which attention can select. Consistent with proposals by D. D. Hoffman and Richards (1984; D. D. Hoffman \& Singh, 1997; see also Biederman, 1987), they suggest that attention can select object parts that are defined on the basis of concave discontinuities of edges. The data also suggest that multiple concave parts can be grouped or organized into a perceptual object that can be processed in parallel. These results are particularly important because they provide new information about both object parts and groups of parts that are available for object-based attentional selection.

\section{EXPERIMENTAL OVERVIEW}

The goal of our experiments was to examine further the nature of the object representations that are available for attentional selection. More specifically, we explored this issue by examining a well-established object-based attentional effect, the same-object effect (i.e., performance is faster and/or more accurate when subjects identify two properties on a single object than one property on one and the other property on another object), within the context of a theoretical framework of perceptual organization suggested by Palmer and Rock (1994b). A graphical representation of this framework is presented in Figure 1. The purpose of this simplified diagram is to illustrate, consistent with the object recognition and perceptual organization literature, the hierarchical nature of object representations that are potentially available for attentional selection.

Clearly, the fact that images can be parsed into their constituent parts on the basis of concave discontinuities is now well established, as is the visual system's ability to apply various grouping principles to recombine object parts into a coherent whole (Biederman, 1987; D. D. Hoffman \& Richards, 1984; D. D. Hoffman \& Singh, 1997; Lowe, 1985; Marr, 1982; Marr \& Nishihara, 1978; Palmer, 1977; Palmer, Rosch, \& Chase, 1981). These representational levels are illustrated in Figure 1 by the parsed and grouped representations, respectively. The panel in the middle of the figure, labeled the Single-UC (uniform connected) Representation, represents a proposal by Palmer and Rock (1994b) that follows from earlier research and arguments by Koffka (1935). Palmer and Rock (1994b, p. 30; see also Palmer \& Rock, 1994a) argue that "the principle of UC is that a connected region of uniform visual properties - such as luminance or lightness, color, texture, motion and possibly other properties as wellstrongly tends to be organized as a single perceptual unit." They further suggest that UC regions are the entry level units in a part-whole hierarchy that can be grouped, on the basis of Gestalt principles, to form larger representations or can be parsed at points of concave discontinuity into smaller representations. They show that UC regions can be perceived as perceptual units even when opposed by powerful grouping principles, such as proximity and similarity.

In the four experiments that we report in the present paper, we employed the theoretical framework illustrated in Figure 1, with single-UC, parsed-UC, and grouped-UC representational levels, as a starting point for the examination of the representations available for object-based attentional selection. More specifically, we employed this theoretical framework to predict when the same-object effect will be obtained (i.e., when subject's performance in identifying two targets will be better when these targets appear on a single object than when one target appears on one object and the other target appears on another object).

The stimuli we employed to address the issue of the representations available for attentional selection differed in an important way from the stimuli used in the majority of previous studies of object-based attentional selection. In previous studies, relatively simple geometric shapes, such as squares, rectangles, polygons, and lines, served as the objects on which targets were located (Baylis \& Driver, 1993; Duncan, 1984; Egly, Driver, \& Rafal, 1994; Egly, Rafal, et al., 1994; Kramer \& Jacobson, 1991; Lavie \& Driver, 1996). Although there are certainly advantages in uti- 


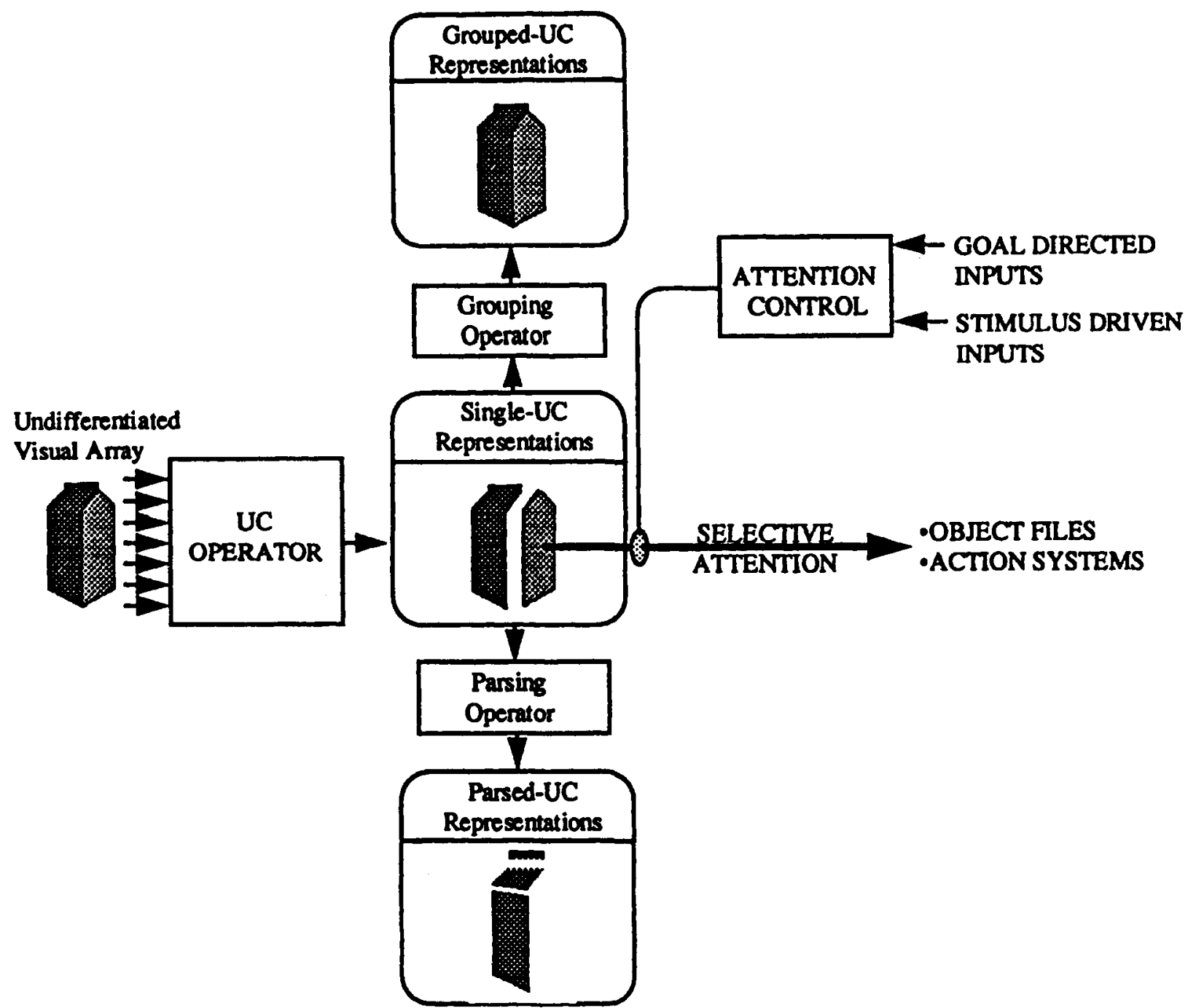

Figure 1. A schematic illustration of Palmer and Rock's (1994b) framework of perceptual organization along with an indication of the potential role of visual attention in selecting different-object representations. The UC operator segregates the incoming visual information into distinct $U C$ regions, which are contiguous regions with homogeneous visual characteristics, such as color, texture, and luminosity. These segregated UC regions are the entry-level representations, where each UC region is a single representation available for attentional selection. The single-UC representations are also available to the grouping operator, which employs classical Gestalt grouping principles (e.g., proximity, similarity, good continuation, common fate, etc.) to form larger grouped-UC representations that are also available for selection. The single-UC representations are also provided to the parsing operator, which segments them at points of concave discontinuity into smaller parsed-UC representations, which are available for selection. In the example, the UC operator segregates the milk carton image into two single-UC representations (a light and a dark gray face) available for independent selection. The two faces are grouped by the grouping operator to recreate the original milk carton image as a single grouped-UC representation available for selection. The parsing operator segments the dark gray faces at points of concave discontinuity on the perimeter, and the resulting three parsed UC representations are available for independent selection. In the illustration, attention is currently directed to a single-UC representation, selecting the light gray face, but it could be directed to the grouped-UC representation level to select the milk carton as a whole or to the parsed-UC representation level to select one of the three smaller parts of the dark gray face.

lizing simple geometric objects to examine a relatively new phenomenon such as object-based attention, the use of isolated and simple geometric shapes and, particularly, shapes in which there are no definable part boundaries (i.e., concave cusps) limits our ability to examine objectbased attentional selection within a part-whole hierarchy. Therefore, we decided to use more complex stimuli that would enable us to systematically manipulate the nature of the parts, in terms of both the number of UC regions that composed an object and the magnitude of the concave discontinuities that define the salience of the parts (D. D. Hoffman \& Singh, 1997). We also decided to use stimuli that would be familiar to our subjects, common everyday wrenches (the "types" of wrenches used in the study can be seen in Figures 2-5). An additional advantage in using such stimuli was that we had the ability to 
Table 1

Mean Response Time (RT; in Milliseconds) and Accuracy (Percent Correct) Values

for the Target-Present Conditions in Experiment 1

\begin{tabular}{|c|c|c|c|c|}
\hline \multirow[b]{2}{*}{ Target Locations } & \multicolumn{2}{|c|}{$\mathrm{RT}(\mathrm{msec})$} & \multicolumn{2}{|c|}{ Accuracy ( $\%$ Correct) } \\
\hline & $M$ & $S E$ & $M$ & $S E$ \\
\hline \multicolumn{5}{|c|}{ Single UC } \\
\hline Same wrench & 879 & 25.3 & 94.9 & 0.5 \\
\hline Different wrenches & 945 & 25.1 & 92.9 & 0.6 \\
\hline \multicolumn{5}{|c|}{ Multiple UC } \\
\hline Same wrench & 903 & 22.4 & 93.9 & 0.5 \\
\hline Different wrenches & 899 & 22.5 & 93.7 & 0.5 \\
\hline
\end{tabular}

determine whether evidence for object-based attentional selection could be generalized from simple geometric shapes to more complex line drawings of everyday objects.

In the four experiments discussed below, we examined whether same-object effects could be obtained for each of the three representational levels illustrated in Figure 1 by manipulating the geometric (i.e., the degree of concave discontinuity) and surface (i.e., the number of UC regions) characteristics of the stimuli; in these experiments, we used pairs of briefly presented wrenches (for an illustration of the stimuli, see the left panels of Figures 2-5). We also examined whether top-down factors could influence the manner in which image-based (bottom-up) properties would be selected. Finally, we examined whether selection from one of the three representational levels would prime selection from the same level of a subsequently presented pair of wrenches, even when the geometric characteristics of this additional wrench pair would bias selection from a different level of the part-whole organizational hierarchy.

\section{EXPERIMENT 1}

Experiment 1 was conducted in order to determine whether single-UC regions can indeed serve as representations from which attention can select. To accomplish this, we had subjects identify whether one or both target properties appeared in a brief display of two wrenches. The target properties, a bent wrench end and an open wrench end (see Figure 2), could appear on one wrench or one property could appear on one wrench and the other property on the other wrench of the briefly presented pair of wrenches. The two properties were located an equivalent distance from each other and were located at a fixed distance from fixation, regardless of whether they appeared on one or on two wrenches. The critical manipulation was whether these target properties would appear on a wrench represented by a single-UC region (i.e., a wrench with a uniform texture) or multiple-UC regions (i.e., a wrench with a textured handle). If subjects can indeed select single-UC regions, we expected a same-object effect for the single-UC but not for the multiple-UC wrench. This follows since each multiple-UC wrench was composed of three $\mathrm{UC}$ regions and, therefore, regardless of whether the target properties were located on one or two wrenches, still required a shift of attention between UC regions.

\section{Method}

Subjects. The subjects were 96 United States Air Force basic trainees, who participated on a voluntary basis during the 6th week of basic training at Lackland Air Force Base, San Antonio. ${ }^{1}$ Eightyfour subjects were male, and 12 subjects were female. The subjects were between 18 and 25 years of age, and all had normal or corrected-to-normal vision.

Apparatus and Stimuli. Stimulus presentation and data collection were conducted with Pentium-based computers equipped with SVGA color monitors and standard QWERTY keyboards. Lefthand responses were made by depressing the D key; right-hand responses were made by depressing the $\mathrm{L}$ key. The subjects were given up to $2 \mathrm{sec}$ to respond. Stimulus presentation was triggered by depressing the space bar. The subjects sat at individual booths in a well-lit room and viewed the stimuli binocularly from a distance of about $62 \mathrm{~cm}$

At the specified viewing distance, when no bent end was present, the wrench display subtended $6^{\circ}$ horizontally and vertically, with a $2.4^{\circ}$ separation between the interior edges of the closest differentobject wrench ends. Each wrench end had a diameter of $1.8^{\circ}$, and the shaft was $0.6^{\circ}$ wide. On presentations where a bent end was present, the bent end was $0.6^{\circ}$ closer to the opposing wrench, reducing the end measurement to $5.4^{\circ}$ and reducing the separation between the two different wrench ends to $1.8^{\circ}$. Wrench ends were centered around a point approximately $3.0^{\circ}$ from fixation. The gap in the open end of the wrench subtended $0.7^{\circ}$ at its minimum separation point. The handle, when present, was $1.5^{\circ}$ in length.

For the single-UC-region wrenches, the entire wrench was filled with light gray (see Figure 2). However, for the multiple-UC-region wrenches, the two wrench ends were light gray and the center of the wrenches was a red-and-blue checkerboard pattern.

Procedure. In each trial, a fixation cross appeared, the subject triggered the display, the stimulus display appeared for $50 \mathrm{msec}$, a blank screen appeared until subject response, then a fixation cross appeared and the next trial began. The subjects searched the display for an open end (shown on the upper right end of examples in Figure 2) and a bent end (shown on the upper left end of the different wrench examples, and the lower right end of the same wrench examples in Figure 2). In each trial, one or both of these targets could appear, and the subjects made one response if only one target was found and the alternative response if both targets were found. The targets could appear on any end, with the exception that when both targets were present, they did not occur on the same end or on diagonal ends. Experimental blocks were balanced such that half of the trials had both targets (i.e., a bent end and an open end) present and half of the trials had only one target (i.e., a bent end or an open end) present.

Each subject performed in a single experimental session. At the beginning of the session, the subjects were presented with computerbased instructions that described and trained them on the task. The subjects completed training when they were able to correctly respond to all eight trials of a training block. Stimuli were presented until the subjects responded in the training block. All subjects were able to successfully complete training with four or fewer blocks of practice.

During the actual experiment, feedback, indicating accuracy, occurred after each experimental block. The subjects performed 384 trials in six 64-trial experimental blocks. The subjects were in. structed to maintain accuracy above $90 \%$ and to respond as quickly as possible.

Design. The experiment was a four-factor design, with response (one or two target properties present), stimulus type (single-UC and 


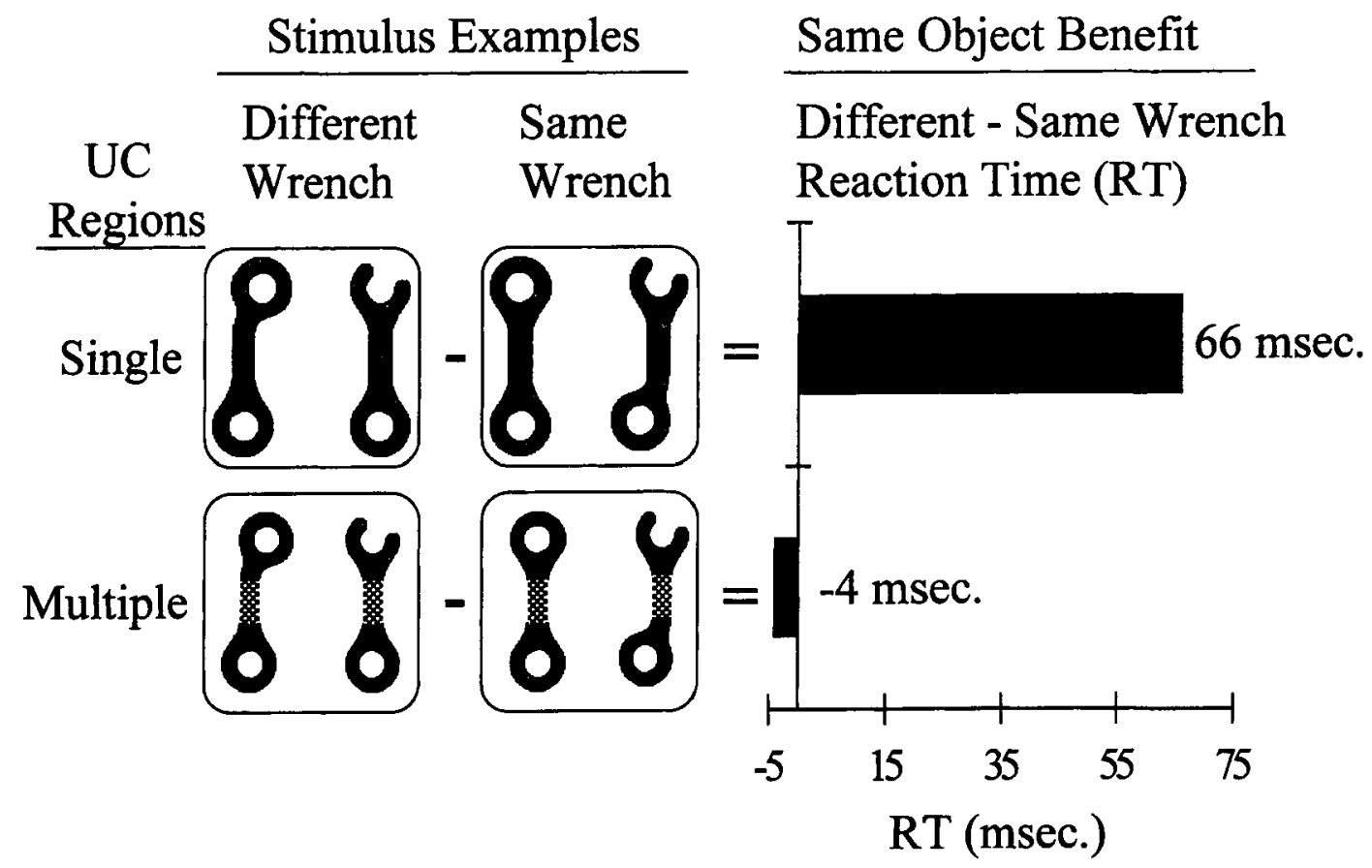

\begin{abstract}
Figure 2. Example stimuli and results from Experiment 1, in which the subjects searched the display for the presence of two targets: an open end (shown on the upper right end of all examples) and a bent end (shown on the upper left end of the different wrench examples, and the lower right end of the same wrench examples). RT differences between same- and different-wrench conditions are presented on the right of the figure and indicate a same-object effect for the single-UC wrench but not for the multiple-UC wrenches.
\end{abstract}

multiple-UC), object (same or different), and orientation (wrenches oriented horizontally or vertically) as factors. Trials were blocked by stimulus type.

\section{Results}

We focus on the trials in which both of the target properties were present, since it is only for these trials that the classification of the same- or different-object condition is defined. Response times (RTs) that were beyond 3 standard deviations ( $S D$ s) from the mean RT of each of the experimental conditions for each subject and RTs from incorrect response trials were excluded from the analysis.

Mean RTs and accuracies are presented in Table 1. An illustration of the displays along with the RT difference between the same- and different-object conditions for the single-UC and multiple-UC stimuli is presented in Figure 2 . Three-way repeated measures analyses of variance (ANOVAs), with stimulus type, object type, and orientation, were performed on the mean RTs and accuracies. A main effect was obtained for the object factor for both RT $[F(1,95)=26.5, p<.01]$ and accuracy $[F(1,95)=$ $13.4, p<.01]$. RTs were faster and accuracies were higher for the same-object trials than for the differentobject trials. More important, however, was the significant two-way interaction between object type and stimulus type for RT $[F(1,95)=5.2, p<.05]$ and accuracy $[F(1,95)=13.0, p<.01]$. As can be seen in Table 1 and Figure 2, same-object trials were responded to more quickly and more accurately than were different-object trials for the single-UC displays, but not for the multipleUC displays. Post hoc comparisons indicated that the difference in RT and accuracy between the same- and different-object trials was significant for the single-UC stimuli, but not for the multiple-UC stimuli. ${ }^{2}$ Neither a main effect nor an interaction of orientation with the other factors was observed.

\section{Discussion}

Previous research has demonstrated that processing two properties on a single object will be fast and/or accurate relative to processing the same two properties on two different objects (Baylis \& Driver, 1993; Duncan, 1984; Kramer et al., 1997; Lavie \& Driver, 1996; Vecera \& Farah, 1994). Indeed, this finding has been taken as strong evidence for object-based attentional selection, since, in the studies in which it has been observed, the critical properties are equidistant regardless of whether they are located on a single object or on multiple objects.

Results from the present experiment were consistent with this observation when both of the target properties (i.e., the open and bent wrench ends) occurred on a singleUC surface, which had homogeneous color, brightness, and texture. In the single-UC condition, the subjects were faster and more accurate to respond when both critical properties were on a single wrench than when they occurred on different wrenches. More importantly, how- 
ever, response speed and accuracy were the same regardless of whether the target properties appeared on one wrench or on two wrenches in the multiple-UC trials.

Thus, the pattern of RTs and accuracies obtained in the single-UC and multiple-UC conditions is consistent with our hypothesis that attention can select representations defined in terms of single-UC regions (Koffka, 1935; Palmer \& Rock, 1994a, 1994b). Within the context of Palmer and Rock's framework of perceptual organization, a same-object effect would be expected for the singleUC wrenches since these stimuli could be selected as a unitary entity. On the other hand, assuming that selection was occurring from UC representations (see the center panel in Figure 1), a same-object effect would not be expected for the multiple-UC wrenches. This follows since each of the two wrenches in the multiple-UC condition was defined by three UC regions: the two ends of the wrenches (which had the same texture and color) and the handle (which was of a different color and texture than the wrench ends). Thus, for the multiple-UC wrenches, two different UC regions needed to be selected whether the target properties appeared on a single wrench or on two different wrenches.

There is, however, an alternative explanation for the pattern of results obtained in the present experiment: Attentional selection was object-based on single-UC trials, whereas selection occurred in a space-based mode on multiple-UC trials. Indeed, the lack of a same-object effect on the multiple-UC trials is consistent with selection via a fixed diameter attentional spotlight, zoom lens, or gradient (Downing \& Pinker, 1985; B. A. Eriksen \& C. W. Eriksen, 1974; C. W. Eriksen \& Yeh, 1985; LaBerge \& Brown, 1989; Posner, 1980). In this case, the distance between target properties, rather than the geometric or surface characteristics, of the stimuli would be the primary factor influencing RT and accuracy. Since the distance between target properties was the same, in our experiment, a space-based model would not predict a difference in performance between same- and different-object trials.

However, we regard the dual-mode selection hypothesis (i.e., object-based selection on single-UC trials and space-based selection on multiple-UC trials) as untenable for several reasons. First, if attention was space based on multiple-UC trials but not on single-UC trials, we would expect spatial priming effects, in the form of faster and more accurate performance when the target properties appeared in the same display location than when they appeared in different locations in subsequent trials, for the multiple-UC condition but not for the single-UC condition (Cave \& Pashler, 1995; J. E. Hoffman \& Nelson, 1981; Kim \& Cave, 1995). However, an analysis of our data failed to reveal spatial priming effects for either the single-UC trials or the multiple-UC trials in the present experiment. ${ }^{3}$ Second, it is not clear why attention would be space based on the multiple-UC trials but objectbased on the single-UC trials, especially since experimental procedures were identical across trials. Lavie and
Driver (1996) had subjects identify two targets on a single object or one target on each of two objects. Selection was object based, as evidenced by faster and more accurate identification when the two targets were located on a single object, on all trials except those on which a single location was precued prior to the presentation of the imperative stimulus. On these trials, there was no difference in performance whether the targets appear on one object or two objects-findings consistent with space-based attentional selection. However, no such precue was employed in the present experiment, and, therefore, it would appear unlikely that space-based attention was selectively employed on the multiple-UC trials. Finally, if attention had been space based on the multiple-UC trials in the present experiment, one would expect a similar pattern of performance - that is, equivalent performance on sameand different-object trials - if the same multiple-UC displays were interspersed among occasional single-wrench displays. However, as will be seen below, the results obtained in Experiment 2 indicate that this was not the case.

\section{EXPERIMENT 2}

Although the results obtained in Experiment 1 suggest that attentional selection took place from single-UC representations, as illustrated in the central panel of Figure 1, we are well aware both from personal experience and from the perceptual organization literature (Biederman, 1987; D. D. Hoffman \& Singh, 1997; Palmer \& Rock, 1994b) that we can perceive stimuli composed of multipleUC regions as coherent objects. For example, we do not usually perceive a motor vehicle as a series of parts (i.e., wheels, side panels, bumpers, etc.) but as a particular model automobile. Therefore, it seems reasonable to assume that subjects should be perfectly capable of selecting, as indicated by a same-object effect, a multiple-UC wrench as a unitary object.

Indeed, given that our stimuli were clearly identifiable as wrenches, regardless of whether they had a handle or not, one may wonder why we failed to find a same-object effect for the multiple-UC stimuli. A possible reason for not obtaining a same-object effect for the multiple-UC wrenches in Experiment 1 concerns the nature of the task. The subjects were required to identify whether two different types of wrench ends were present in a briefly presented display. The handles were not relevant for these judgments and indeed might have interfered with the task that the subjects were instructed to perform. Therefore, it appears conceivable that, rather than treating the multiple-UC wrenches as a single entity, at the level of a grouped representation, instead the wrench ends would be selected from the available single-UC representations. Such a strategy would result in a same-object effect for the single-UC wrench but not for the multiple-UC wrenches. However, an alternative possibility is that the 50 -msec presentation duration was insufficient to construct a grouped representation, and, therefore, there was no option but to 


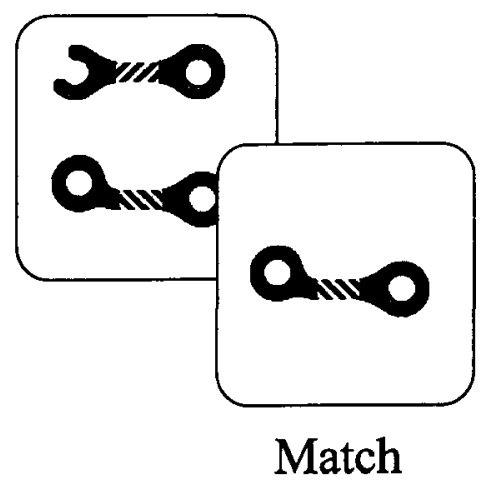

\section{Same Object Benefit}
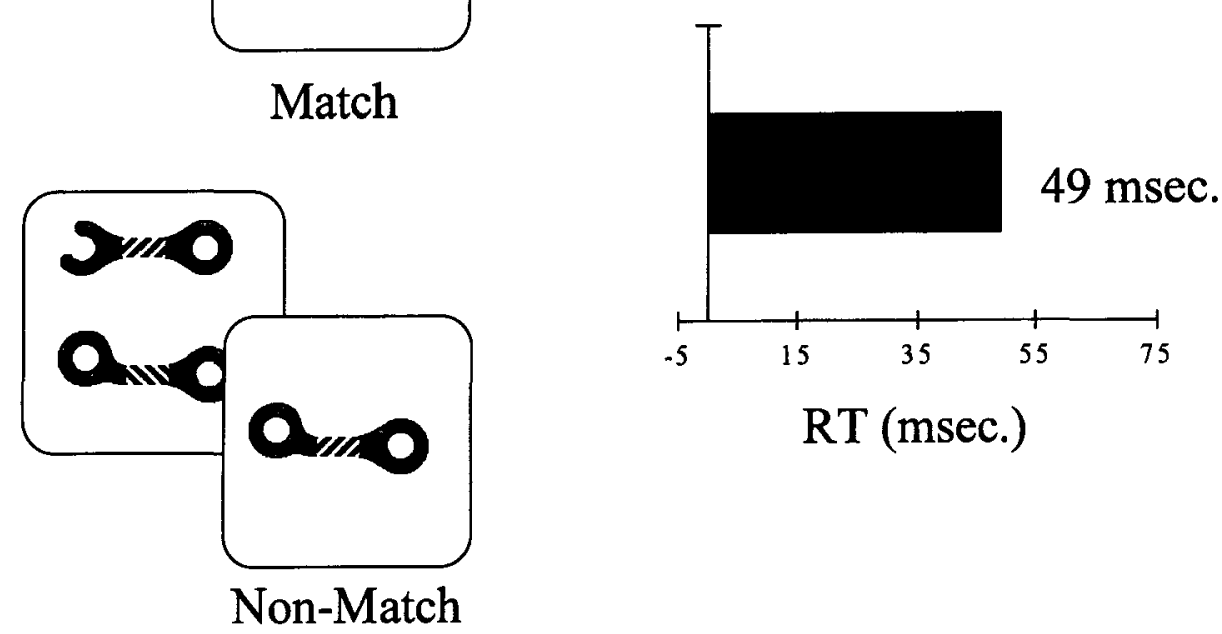

Figure 3. Example stimuli and results from Experiment 2. The paradigm was essentially the same as Experiment 1, except that, on $20 \%$ of the trials, after the subjects responded to the target search task a probe stimulus appeared that was to be compared with the preceding presentation. Shown are examples of trials in which the correct probe response was a match (both ends and handle match the bottom wrench) and a trial in which the correct response was a nonmatch (the handle matches the top wrench, the ends match the bottom wrench). Results demonstrate that the probe task, which required the subjects to process the entire wrench, was successful in encouraging a 49-msec sameobject effect for the target search task.

select the wrench ends from the UC representations, thereby resulting in a failure to observe a same-object effect for the multiple-UC wrenches.

In Experiment 2, we required subjects to occasionally decide whether a single probe wrench, which followed the presentation of a pair of wrenches that were to be judged in terms of their ends as in Experiment 1, matched one of the previously presented wrenches. The probe wrench could either match one of the two wrenches or be composed of the handle from one of the previously presented wrenches and the ends of the other wrench, in which case a mismatch response would be required. Thus, what we hoped to achieve by implementing this occasional probe task was to encourage subjects to select the wrenches as unitary objects, despite the fact that they were composed of multiple-UC regions. Our assumption was that subjects would be more likely to select the wrenches as grouped multiple-UC regions than as single-UC regions, as the data suggest was the case in Experiment 1, if, on a number of random trials, they would be required to indicate whether the three UC regions that composed a single wrench had been presented together or not. That is, we assumed that the processing demands of the occa- sional secondary probe task would influence the manner in which the briefly presented wrenches were attended.

On the basis of these assumptions, we expected that a same-object effect would be obtained for the multipleUC wrenches - that is, exactly the same wrenches that failed to produce a same-object effect in Experiment 1. Within the context of the part-whole organizational hierarchy illustrated in Figure 1, we would interpret such an effect as a shift from selection at the single-UC representational level (i.e., the central panel of the figure) to the grouped representational level (i.e., the upper panel in the figure).

\section{Method}

Subjects. The subjects were 89 United States Air Force basic trainees, who participated on a voluntary basis. Fifty-nine subjects were male, and 30 subjects were female. The subjects were between 18 and 28 years of age, and all had normal or corrected-to-normal vision. None of the subjects had participated in any of the other experiments described in the present paper.

Apparatus and Stimuli. Stimuli used in Experiment 2 are illustrated in Figure 3. The computer systems, stimuli, and responses employed in the present experiment were the same as those used in Experiment 1, with the following exceptions. Displays for the pri- 
mary task - the same task that the subjects performed in Experiment 1-consisted of two main geometric figures, which resembled a pair of wrenches with striped handles covering the majority of their shafts. The stripes (presented in red and blue) on the handles of the two wrenches always had opposite orientations. The critical information for the performance of the primary task occurred at any of the four ends of the wrenches and occurred as an open-end and a bent-end for the target-present conditions or as either an openend or a bent-end for the target-absent response.

Displays for the secondary task, which occurred on a random $20 \%$ of the total trials, consisted of a single wrench constructed from one or both of the wrenches presented in the primary task display. In $50 \%$ of the secondary task trials, the probe wrench was the same as one of the primary task wrenches. In the other $50 \%$ of the secondary task probe trials, the wrench was composed of the handle from one of the primary task wrenches and the ends from the other primary task wrench. The subjects' task was to press one response button if the probe wrench matched one of the two primary task wrenches and to press the other response button if the probe wrench was composed of parts from the two primary task wrenches.

Procedure. The procedure was identical to that employed in Experiment 1, with the following exceptions. As in Experiment 1, a pair of wrenches was presented for $50 \mathrm{msec}$, after which the subjects were given $2,000 \mathrm{msec}$ to decide whether or not both of the critical properties were present. After $80 \%$ of the trials, a fixation cross reappeared, which served as a cue to indicate to the subjects that they could begin the next trial by depressing the space bar on the computer keyboard. However, on $20 \%$ of the trials, a secondary task probe display would appear for $50 \mathrm{msec}$ with a single wrench, and the subjects were required to indicate whether this wrench was one of the two wrenches that they had seen in the previous display. Following the subject's response, a fixation cross would reappear, and the subject could begin the next trial by depressing the space bar.

The subjects performed in a single experimental session that lasted approximately $1 \mathrm{~h}$. At the beginning of the session, the subjects were presented with computer-based instructions that described and trained them on the task. The subjects completed training when they were able to correctly respond to 8 primary task trials and 2 probe trials of a training block. Stimuli were presented until the subjects responded in the training block. All subjects were able to successfully complete training with five or fewer blocks of practice. The subjects performed 420 experimental trials: 336 in which only the primary task was performed, and 84 in which both the primary and the secondary task were performed.

Design. The experiment was a three-factor design, with object type (same and different), orientation (vertical and horizontal), and response (targets present or absent) as within-subjects factors. On the probe trials, the single wrench occurred equally often with each of the four stimulus and two response types.

\section{Results}

We focus on the trials in which both of the target properties were present, since it is only for these trials that the classification of the same- or different-object condition is defined. RTs that were beyond $3 S D$ s from the mean RT of each of the experimental conditions for each subject and RTs from incorrect response trials were excluded from the analysis.

An illustration of the displays along with the RT difference between the same- and different-object conditions is presented in Figure 3. Two-way repeated measures ANOVAs, with object type and orientation as factors, were performed on the mean RTs and accuracies. A main effect was obtained for the object factor for both RT
$[F(1,88)=44.7, p<.01]$ and accuracy $[F(1,88)=11.7$, $p<.01]$. RTs were faster ( 846 and $895 \mathrm{msec}$ for the same- and different-object trials, respectively) and accuracies were higher $(92.5 \%$ and $89.7 \%$ for the same- and different-object trials, respectively) for the same-object trials than for the different-object trials. There was no significant main effect for orientation or interaction of orientation with object type.

\section{Discussion}

The results were quite straightforward. When the multiple-UC wrenches were in a context in which they were occasionally followed by a single wrench probe, same-object trials were faster and more accurate than different-object trials. This result stands in marked contrast to the results obtained in Experiment 1 with the multiple-UC wrench display, in which a same-object effect was not obtained.

Indeed, these results suggest that the level of representation at which objects are attended is quite flexible and depends on the demands of the task and the context in which the task occurs. In Experiment 1, the subjects were required only to decide whether a bent end and an open end were present in the display. Whether a handle was present or not on the wrenches was irrelevant. On the other hand, although the presence of a handle was also irrelevant for the bent-end/open-end decision in Experiment 2 , the occurrence of the secondary probe wrench, which did require knowledge of the relationship among the wrench ends and handle, was unpredictable. Therefore, it was to the subjects' advantage to attend to the multipleUC regions that defined the wrench as a unitary perceptual object rather than to attend to single- $\mathrm{UC}$ regions.

In some respects, these results (i.e., the absence or presence of the same-object effect for the multiple-UC wrenches in Experiments 1 and 2) are similar to the results of previous studies that have reported top-down effects on object-based attentional selection. For example, Yantis (1992) reported that subjects were more accurate in tracking five continuously moving target dots among five moving distractor dots if they had been told to interpret the target dots as vertices of rigid or nonrigid objects. Yantis interpreted these results as evidence that subjects were capable of grouping randomly moving dots into a virtual object that was easier to track than five isolated dots. In a similar vein, Baylis and Driver (1993; see also Baylis, 1994) obtained a same-object effect for contour judgments on ambiguous figure-ground displays when subjects were told to imagine the contours to be on a single object. When subjects were instructed to imagine that the same two contours were on two different objects, a same-object effect was not obtained. Thus, the Yantis (1992) and Baylis and Driver (1993) results suggest that top-down factors, in the form of expectancies and instructions, are sufficient to encourage object-based attentional selection. The results obtained in Experiments 1 and 2 are compatible with this conclusion. However, the 
results of our experiments extend previous findings by suggesting that top-down factors-in the present case, the context in which the property identification task was performed-can encourage a shift in the representational level, from the single-UC to the grouped-level representation, as illustrated in Figure 1, from which objects are selected. ${ }^{4}$

\section{EXPERIMENT 3}

The results of Experiments 1 and 2 suggest that objectbased attentional selection can occur from at least two different representational levels: from single-UC representations as suggested by the results obtained in Experiment 1 and from grouped representations as suggested by the results obtained in Experiment 2. According to Palmer and Rock's (1994b) theoretical framework illustrated in Figure 1, it appears conceivable that object-based attentional selection may also occur from parsed representations. In an effort to investigate whether this is indeed the case, we examined, in Experiment 3, the extent to which changes in the geometric characteristics of the wrenches will promote a shift in attentional selection from singleUC to parsed representations.

As previously discussed, there is now relatively wide agreement that objects are often parsed into parts at concave discontinuities and that these parts serve as the basis for object recognition, particularly when the objects are degraded or partially occluded (Biederman, 1987; D. D. Hoffman \& Richards, 1994; Marr, 1982; Marr \& Nishihara, 1978). Indeed, D. D. Hoffman and Singh (1997) suggested that one of the important factors in determining the salience of object parts is the magnitude of the curvature that defines the concave discontinuities in the boundaries of objects. They supported this proposal with a number of visual demonstrations and the results from a series of psychophysical experiments.

In the present experiment, we systematically increased the magnitude of the curvature at the point at which the wrench ends connect to the shaft in order to examine whether increased salience of the wrench parts (i.e., the two ends and the shaft) would result in a shift of objectbased attentional selection from the single UC to the parsed representations. Graphical representations of the wrench pairs employed in Experiment 3 are illustrated in Figure 4. As can be seen in the figure, the magnitude of the concave discontinuity systematically decreases from the wrench pairs at the top of the figure to the wrench pairs at the bottom of the figure. The wrench pairs in the center of the figure provide an anchor point to our previous experiments, since this pair is identical to the wrenches used in Experiments 1 and 2.

If changes in the magnitude of the concave discontinuities do indeed encourage a shift in object-based attentional selection from the single $\mathrm{UC}$ to the parsed representations, then we would expect systematic decreases in the size of the same-object effect with increases in the magnitude of the curvature that defines the point of concave discontinu- ity at which the wrench ends are connected to the shaft. This follows since selection from parsed representations will entail a need to shift attention between wrench ends regardless of whether the target wrench ends are located on one or two wrenches. That is, since the wrench ends (and wrench shafts) are represented as individual entities at the parsed representational level, there will be no benefit (as reflected in a same-object effect) for locating the target properties on the same wrench. Alternatively, it is conceivable that object-based attention selects the wrench as a single-UC region and that parsing is then subsequently carried out on the basis of the magnitude of the concave discontinuity at the point at which the wrench ends are attached to the shaft. In such a case, we would not expect any change in the size of the same-object effect with changes in the magnitude of the concave discontinuity.

\section{Method}

Subjects. The subjects were 89 United States Air Force basic trainees, who participated on a voluntary basis. Fifty-six subjects were male, and 33 subjects were female. The subjects were between 18 and 30 years of age, and all had normal or corrected-to-normal vision. None of the subjects had participated in any of the other experiments described in the present paper.

Apparatus and Stimuli. Stimuli used in this experiment are illustrated in Figure 4. The computer systems, stimuli, and responses employed in the present experiment were the same as those used in Experiment 1, with the following exceptions. Three different versions, defined in the terms of the extent of the concave discontinuity at the point that the wrench ends attached to the wrench shafts, of the single-UC wrenches that were employed in Experiment 1 were used in this experiment. The wrenches are ordered in Figure 4, from the top left to the bottom left of the figure, in terms of the decreasing concavity where the wrench ends meet the shaft. The wrenches represented in the central panels in Figure 4 were identical to the wrenches employed in Experiments 1 and 2.

Procedure. The procedure was identical to that employed in Experiment 1.

Following practice, the subjects performed 384 experimental trials, in six 64-trial blocks. Stimulus type served as a blocking factor, with two blocks performed for each of the three stimulus types. The order of the blocks was counterbalanced across subjects.

Design. The experiment was a four-factor design, with object type (same and different), stimulus type (good-parsing point, poorparsing point, and no-parsing point wrenches), orientation (vertical and horizontal), and response (targets present or absent) as withinsubjects factors.

\section{Results}

We focus on the trials in which both of the target properties were present, since it is only for these trials that the classification of the same- or different-object condition is defined. RTs that were beyond $3 S D$ s from the mean RT of each of the experimental conditions for each subject and RTs from incorrect response trials were excluded from the analysis.

Mean RTs and accuracies are presented in Table 2. An illustration of the displays along with the RT difference between the same- and different-object conditions is presented in Figure 4. Three-way repeated measures ANOVAs, with stimulus type, object type, and orientation, were performed on the mean RTs and accuracies. A significant 


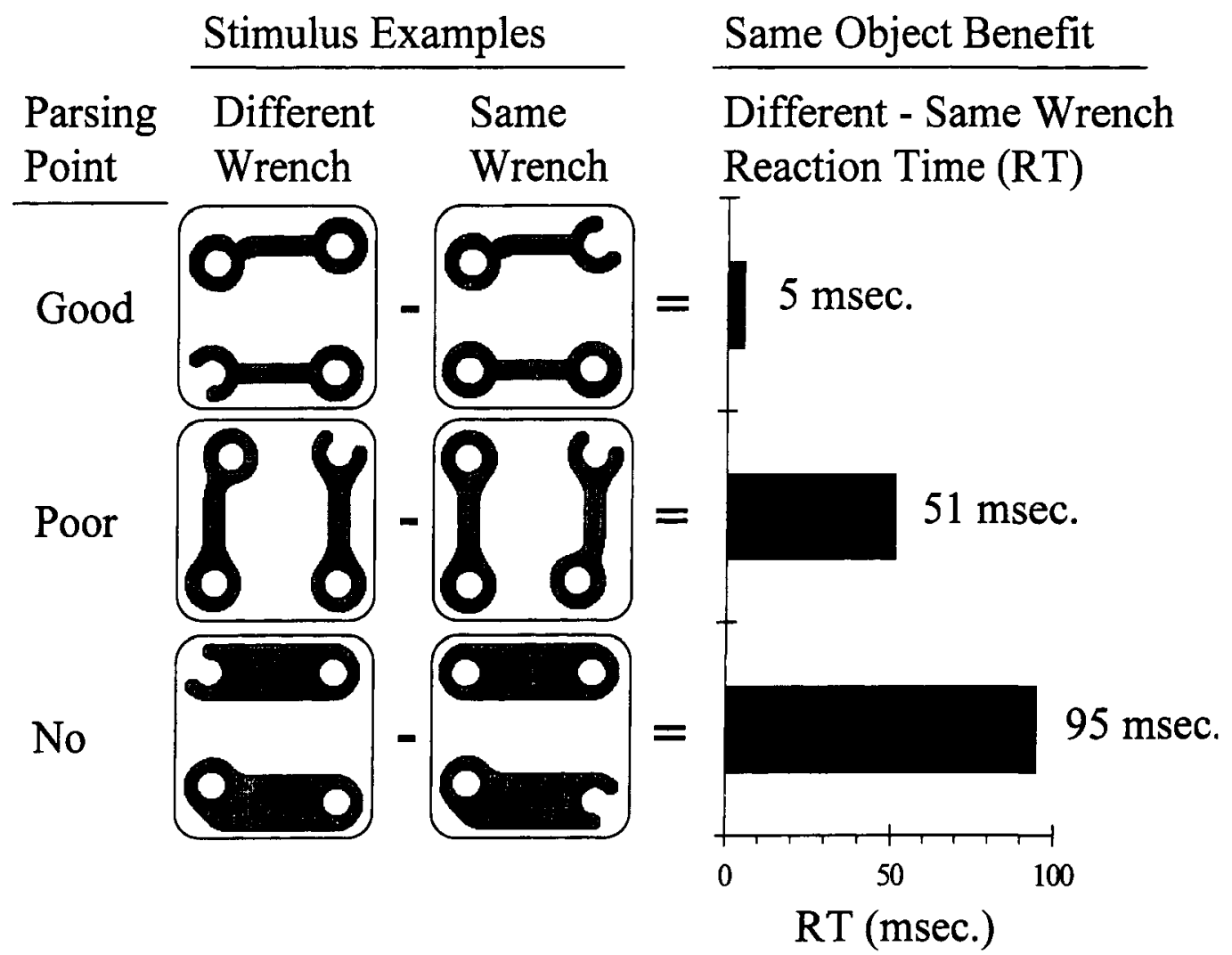

\begin{abstract}
Figure 4. Example stimuli and results from Experiment 3. The paradigm was identical to that of Experiment 1, with the exception of the appearance of the stimuli. The perimeter of the basic stimuli contained good-parsing points (sharp concavities), poor-parsing points (smooth concavities), or no-parsing points (no concavities). The same-object RT effect was largest for the no-parsing point stimuli ( $95 \mathrm{msec}$ ), smaller for the poor-parsing point stimuli (51 msec), and effectively nonexistent for the good-parsing point stimuli $(5 \mathrm{msec})$.
\end{abstract}

main effect was obtained for object type for $\mathrm{RT}[F(1,88)=$ $26.7, p<.01]$ and accuracy $[F(1,88)=6.4, p<.01]$. RTs were faster and accuracies were higher for the sameobject trials than for the different-object trials. More important, however, was the significant two-way interaction for object and stimulus type for RT $[F(2,176)=10.1, p<$ $.01]$ and accuracy $[F(2,176)=3.0, p<.05]$. As illustrated in Figure 4 and Table 2, the difference in RT and accuracy between same- and different-object trials increased from the good-parsing to the poor-parsing to the no-parsing point displays. Post hoc comparisons indicated that these differences were significant for the poor- and no-parsing point wrenches, but not for the good-parsing point wrenches. No significant effects were obtained for orientation.

\section{Discussion}

As predicted on the basis of previous proposals that object parts increase in salience with increases in the magnitude of concave discontinuities of object boundaries (D. D. Hoffman \& Singh, 1997), the magnitude of the same-object effect increased with decreases in magnitude of the concave cusps at which the wrench ends were connected to the shafts. Thus, the present data, like those obtained in Experiment 1 in which surface characteristics of the wrenches were varied, suggest that the nature of the representation that object-based attention selects can be influenced by image-based (i.e., bottom-up) properties of the display. The findings of the Experiments 1-3 also suggest, consistent with the theoretical framework illustrated in Figure 1, that object-based attentional selection, as reflected by the now classic same-object effect (Baylis \& Driver, 1993; Duncan, 1984; Lavie \& Driver, 1996; Vecera \& Farah, 1994), can occur from at least three different representational levels: single-UC representations, grouped representations, and parsed representations.

However, although we have now demonstrated, using both surface characteristics (Experiment 1) and geometric properties of objects (Experiment 3 ), the influence of image-based properties on object-based attentional selection among the three hypothesized representational levels, we have provided only a single demonstration of the influence of top-down factors on object-based selection. In Experiment 2, we demonstrated that, when the property identification task was embedded within the context of a secondary task in which the wrench ends were to be related to the wrench handle, the subjects selected the multiple-UC wrenches as a single entity. This conclusion was supported by the observation of the same-object ef- 
Table 2

Mean Response Time (RT; in Milliseconds) and Accuracy (Percent Correct) Values

for the Target-Present Conditions in Experiment 3

\begin{tabular}{|c|c|c|c|c|}
\hline \multirow[b]{2}{*}{ Target Locations } & \multicolumn{2}{|c|}{$\mathrm{RT}(\mathrm{msec})$} & \multicolumn{2}{|c|}{ Accuracy ( $\%$ Correct) } \\
\hline & $M$ & $S E$ & $M$ & $S E$ \\
\hline \multicolumn{5}{|c|}{ Good-Parsing Point Wrenches } \\
\hline Same wrench & 808 & 17.1 & 95.7 & 0.9 \\
\hline Different wrenches & 813 & 17.9 & 95.4 & 0.9 \\
\hline \multicolumn{5}{|c|}{ Poor-Parsing Point Wrenches } \\
\hline Same wrench & 813 & 18.9 & 95.9 & 0.7 \\
\hline Different wrenches & 864 & 16.5 & 92.0 & 0.6 \\
\hline \multicolumn{5}{|c|}{ No-Parsing Point Wrenches } \\
\hline Same wrench & 794 & 15.6 & 97.4 & 0.6 \\
\hline Different wrenches & 889 & 18.6 & 96.4 & 0.7 \\
\hline
\end{tabular}

fect for the multiple-UC wrenches in Experiment 2 (i.e., in contrast to the lack of a same-object effect for the multiple-UC wrenches in Experiment 1 in which the secondary probe task was not employed). In Experiment 4, we examined whether the manipulation of experimental context could be employed to promote selection of the good-parsing point wrenches (see Figure 4) from the UC representational level rather than from the parsed representational level, as we argued was the case in Experiment 3. That is, we examined whether a same-object effect would be observed for the good-parsing point wrenches when they were embedded in a context that would encourage selection from single-UC representations.

\section{EXPERIMENT 4}

In the Experiment 4, we employed a paradigm developed by Ward (1982) to prompt subjects to select the good-parsing point wrenches from the single-UC representational level. Ward demonstrated that the attentional system displays a robust "level readiness effect" when subjects must select task-critical information from either a local or a global level of compound letter stimuli (i.e., stimuli popularized by Navon, 1977, in which large letters are composed of small letters). Similar to the twostate switching model of Sperling and Melcher (1978; see also Johnson \& Yantis, 1995), Ward proposed that attention can switch between global and local representations (see also J. E. Hoffman, 1980; Kinchla, Solis-Macias, \& J. E. Hoffman, 1983). If task-critical attributes occur at the level that attention is directed to, responses will be fast and accurate. On the other hand, if task-critical attributes occur at the unattended level, it takes time to redirect attention, thereby slowing responses and increasing the probability of error.

Ward (1982) investigated the level readiness idea by presenting subjects with two compound letters in rapid succession (see also Robertson, 1996; Robertson, Egly, Lamb, \& Kerth, 1993). The first presentation was intended to prime attention to a given level within the global-local hierarchy, and the effects of priming this level were expected to be evidenced in the performance on the subsequent probe presentation. Each of these prime-probe couplets composed a single trial. Trials were blocked such that subjects were instructed to judge the critical form (i.e., the identity of the large or the small letters) in one of four orders: global/global (GG), local/local (LL), global/local (GL), and local/global (GL).

Data from the probe trials supported the level readiness hypothesis: Performance was better in the GG condition than in the LG condition, and performance was better in the LL condition than in the GL condition. That is, responses were faster and more accurate when the probe's critical attribute was at the same level as the prime's critical attribute, so that attention did not need to be switched between the local and global levels of the stimuli. This level readiness, or level priming, effect was obtained even for delays of up to $2.5 \mathrm{sec}$ between stimulus presentations. Ward (1982) interpreted these data as evidence that attention remained focused at a particular level of the global-local hierarchy for a short period of time after the selection of a stimulus.

We employed Ward's (1982) paradigm in Experiment 4 with the good-parsing $(G)$ and no-parsing $(N)$ point wrenches from Experiment 3 (see Figures 4 and 5) in an effort to examine the extent to which level readiness effects could be used to prime selection from the single-UC representational level. Our logic was as follows: Since, as suggested by the results of Experiment 3, the no-parsing point wrenches are selected from the single-UC representational level, then a good-parsing point wrench part (i.e., wrenches that are selected from the parsed representational level as suggested by the results of Experiment 3) that is preceded by a no-parsing point wrench (i.e., N/G) will also be selected from the single-UC representational level. That is, a same-object effect will be obtained for the good-parsing point wrenches in the $\mathrm{N} / \mathrm{G}$ sequence but not in the G/G sequence. Of course, this prediction is predicated on the assumption that parsing of objects into parts at concave discontinuities is optional, at least with regard to object-based attentional selection. ${ }^{5}$

What should we expect for the G/N sequence? Clearly, the results of Experiment 3 suggested that the goodparsing point wrenches were selected from the parsed representations, and, therefore, we might expect that the no-parsing point wrenches would also be primed to be selected at this level of the organizational hierarchy. However, it is also the case that there is little support for parsing of the no-parsing point wrenches given the lack of concave discontinuities in the contours of these stimuli. Therefore, it seems unlikely that the $\mathrm{G} / \mathrm{N}$ sequence will lead to a change in the level at which the no-parsing point wrenches are selected relative to the $\mathrm{N} / \mathrm{N}$ sequences. In other words, we predict that a same-object effect will be obtained for the no-parsing point wrenches, indicating selection from the single-UC representational level, in the $\mathrm{N} / \mathrm{N}$ and $\mathrm{G} / \mathrm{N}$ sequences. 


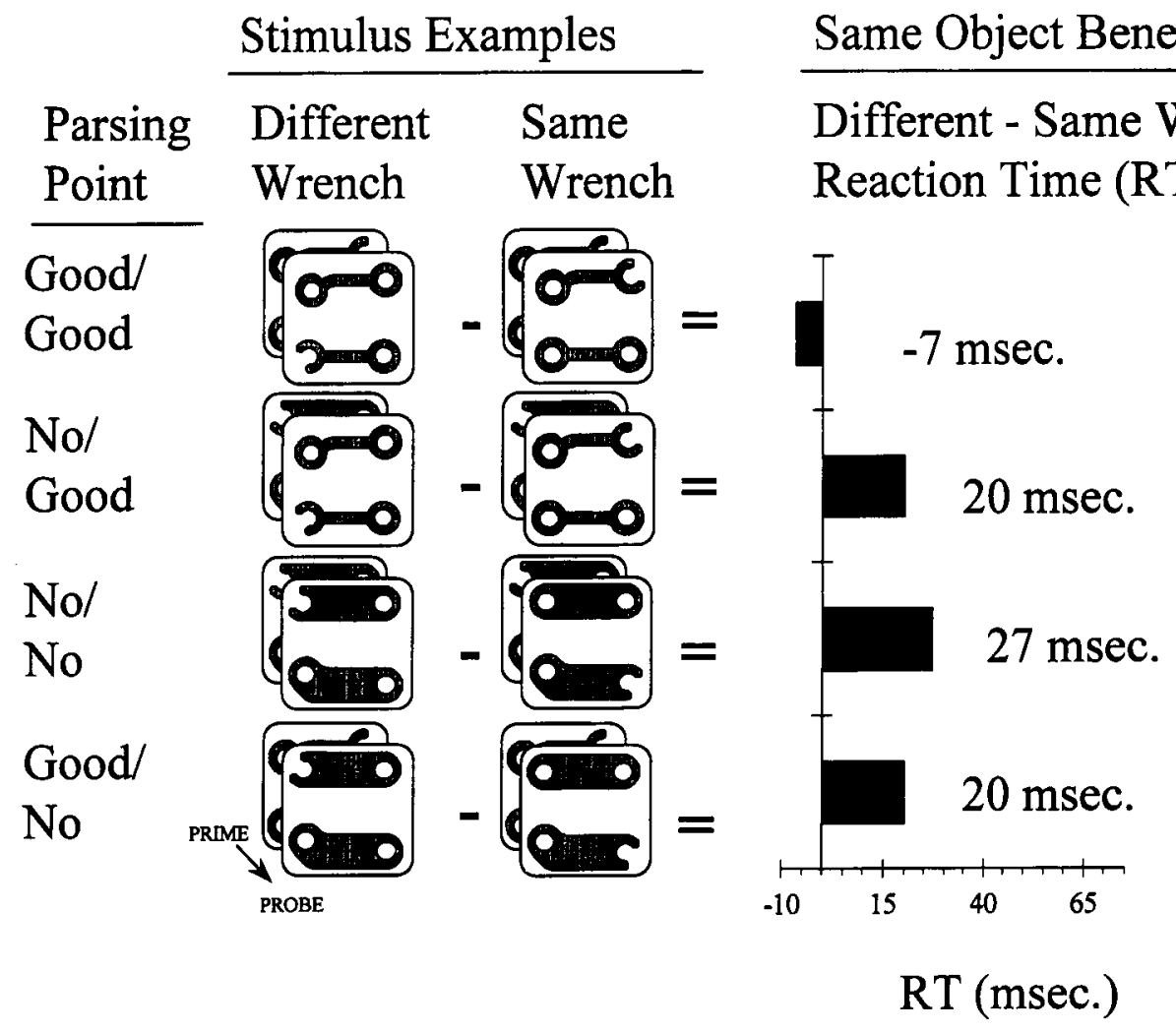

Figure 5. Example stimuli and results from Experiment 4. The target search paradigm was the same as Experiments 1-3, with the exception that, on $75 \%$ of the trials, a prime stimulus (shown in the gray frames) preceded the probe stimulus (shown in the black frames). Results demonstrate that when the good-parsing point stimuli were preceded by good-parsing point stimuli (the good/good condition), a same-object RT effect was not demonstrated, replicating Experiment 3; in contrast, when good-parsing point stimuli were preceded by no-parsing point stimuli (the no/good condition), a same-object RT effect was obtained for the good-parsing point stimuli.

\section{Method}

Subjects. The subjects were 90 United States Air Force basic trainees, who participated on a voluntary basis. Eighty-six subjects were male and 4 subjects were female. The subjects were between 18 and 25 years of age, and all had normal or corrected-to-normal vision. None of the subjects had participated in any of the other experiments described in the present paper.

Apparatus and Stimuli. Stimuli used in this experiment are illustrated in Figure 5. These wrench pairs were identical to the goodparsing and no-parsing point stimuli employed in Experiment 3 (see Figure 4).

Procedure. The experiment was performed in a single 1-h session. The session consisted of 12 experimental blocks of 40 trials each, with feedback indicating accuracy for single- and dualpresentation trials separating the blocks. Training for the singlepresentation trials (i.e., a single display of two wrenches) was done as in the previous experiments and was followed by training for dual presentation (two displays of two wrenches each in succession). In the dual-presentation training blocks, the subjects were required to attain $100 \%$ accuracy on blocks of 15 trials, 3 of which were single presentation and 12 of which were dual presentations.

For single-presentation trials, the subjects were instructed to fixate a small white cross that appeared at the center of the display and to depress the space bar to begin the trial when ready. The stimulus appeared for $50 \mathrm{msec}$, and then a blank screen was presented. After $50 \mathrm{msec}$, a $500-\mathrm{Hz}$ tone sounded for $100 \mathrm{msec}$, instructing the subject to respond to the single presentation. If an incorrect response was entered, or if no response was entered within $3,000 \mathrm{msec}$ after the aural cue, a $50-\mathrm{Hz}$ tone sounded for $100 \mathrm{msec}$; if a correct response was entered, no tone sounded. At this point, a white fixation cross appeared, signifying that the subject could begin the next trial. The single-presentation trials were included in the experiment in order to encourage the subjects to attend to the prime display on the dual-presentation trials.

Dual-presentation trials began exactly as single-presentation trials, so the subjects would have no indication which type of trial was being performed: The white fixation cross signifying the beginning of the trial appeared until the space bar was depressed, and the prime stimulus appeared for $50 \mathrm{msec}$. Then, after $1,000 \mathrm{msec}$, a second stimulus appeared for $50 \mathrm{msec}$. After this probe stimulus disappeared, a blank screen was presented, and, $50 \mathrm{msec}$ later, a 500 $\mathrm{Hz}$ tone sounded for $100 \mathrm{msec}$, which instructed the subject to respond to the probe stimulus. Either after the subject responded or after 3,000 msec elapsed without a response, a dark gray fixation cross was presented, and a $50-\mathrm{Hz} 100-\mathrm{msec}$ tone sounded if either no response or an incorrect response had been entered. After $5,500 \mathrm{msec}$ from the presentation of the prime display, the dark fixation cross changed to white, indicating that the subject could begin the next trial by depressing the space bar.

The experimental trials were divided into four trial-type blocks $(\mathrm{G} / \mathrm{G}, \mathrm{N} / \mathrm{G}, \mathrm{N} / \mathrm{N}$, and $\mathrm{G} / \mathrm{N}$ ) of 120 trials per block. The presentation of the order of the blocks was counterbalanced across subjects. Each stimulus display of two wrenches could be from either the goodparsing point set $(\mathrm{G})$ or from the no-parsing point $(\mathrm{N})$ set from $\mathrm{Ex}$ - 
Table 3

Mean Response Time (RT; in Milliseconds) and Accuracy (Percent Correct) Values for the Target-Present Conditions in Experiment 4

\begin{tabular}{|c|c|c|c|c|}
\hline \multirow[b]{2}{*}{ Target Locations } & \multicolumn{2}{|c|}{ RT (msec) } & \multicolumn{2}{|c|}{ Accuracy $(\%$ Correct $)$} \\
\hline & $M$ & $S E$ & $M$ & $S E$ \\
\hline \multicolumn{5}{|c|}{ G/G Trial Sequence } \\
\hline Same wrench & 886 & 20.1 & 96.7 & 0.5 \\
\hline Different wrenches & 879 & 20.5 & 97.5 & 0.4 \\
\hline \multicolumn{5}{|c|}{ G/N Trial Sequence } \\
\hline Same wrench & 884 & 20.4 & 98.0 & 0.5 \\
\hline Different wrenches & 904 & 21.3 & 97.0 & 0.5 \\
\hline \multicolumn{5}{|c|}{ N/G Trial Sequence } \\
\hline Same wrench & 882 & 20.7 & 97.1 & 0.7 \\
\hline Different wrenches & 902 & 20.8 & 97.0 & 0.6 \\
\hline \multicolumn{5}{|c|}{ N/N Trial Sequence } \\
\hline Same wrench & 864 & 19.2 & 98.4 & 0.3 \\
\hline Different wrenches & 891 & 20.4 & 98.3 & 0.4 \\
\hline
\end{tabular}

Note $-\mathrm{G}=$ good-parsing point set; $\mathrm{N}=$ no-parsing point set.

periment 3 (see Figure 4). The four possible prime-probe relationships, therefore, defined the trial type for each of the four blocks: $\mathrm{G} / \mathrm{G}, \mathrm{N} / \mathrm{G}, \mathrm{N} / \mathrm{N}$, and $\mathrm{G} / \mathrm{N}$. Within each of the trial blocks, 8 trials were single-presentation trials and 32 were dual-presentation trials. For the 8 single-presentation trials, $50 \%$ of the trials were targetpresent trials (i.e., both the open end and the bent end were present) and $50 \%$ of the trials were target-absent trials (i.e., either the open end or the bent end was present). In the target-present trials, half of the trials were same-object trials and the other half were different-object trials, $50 \%$ of each of these two types of trials were presented vertically, and the other $50 \%$ were presented horizontally. For the dualpresentation trials, all combinations of object type, orientation, and response type were represented for the prime and probe sequences.

Design. The experiment was a four-factor design, with object type (same and different), trial type (G/G,N/G,N/N, and $G / N)$, orientation (vertical and horizontal), and response (targets present or absent) as within-subjects factors.

\section{Results}

We focus on the trials in which both of the target properties were present, since it is only for these trials that the classification of the same- or different-object condition is defined. RTs that were beyond $3 S D$ s from the mean RT of each of the experimental conditions for each subject and RTs from incorrect response trials were excluded from the analysis.

Mean RTs and accuracies are presented in Table 3. An illustration of the displays along with the RT difference between the same- and different-object conditions for the $\mathrm{G} / \mathrm{G}, \mathrm{N} / \mathrm{G}, \mathrm{N} / \mathrm{N}$, and $\mathrm{G} / \mathrm{N}$ trial sequences is presented in Figure 5. Three-way repeated measures ANOVAs, with trial type, object type, and orientation, were performed on the mean RTs and accuracies. A main effect was obtained for object type $[F(1,89)=5.8, p<.01]$. RTs were faster $(879 \mathrm{msec})$ when both of the target properties were located on one wrench than when one target property was located on each of the two wrenches $(894 \mathrm{msec})$. More important, however, was the significant two-way interaction between object type and trial type $[F(2,267)=$ $3.5, p<.05]$. Consistent with the results of Experi- ment 3 , post hoc comparisons indicated significantly faster performance on the same-object trials than on the different-object trials for the N/N stimuli, but not for the $\mathrm{G} / \mathrm{G}$ stimuli. Even more interesting was the finding that, although no same-object effect was obtained for the goodparsing point stimuli in Experiment 3 or in the G/G trials in Experiment 4, a same-object effect was observed for the good-parsing point stimuli when these stimuli were preceded by a no-parsing point prime display (i.e., N/G). Thus, it would appear that, in some cases, a prior selection episode at one level of object representation (i.e., from UC representations; see Figure 1) can encourage the selection of subsequent objects at the same level of representation, even when these objects are often selected at other representational levels (e.g., the good-parsing point stimuli selected as a single UC region).

Accuracies were very high in Experiment 4 . None of the accuracy main effects or interactions were statistically significant.

\section{Discussion}

The most important result obtained in Experiment 4 was the finding of a same-object effect, as indicated in Figure 5 and Table 3 , for the good-parsing point wrenches in the $N / G$ sequence. The same good-parsing point wrenches that failed to produce a same-object effect in Experiment 3 and in the $\mathrm{G} / \mathrm{G}$ sequence in Experiment 4 produced a same-object effect when preceded by a goodparsing point wrench. These results suggest that (1) parsing at points of concave discontinuity is optional, at least with regard to object-based attentional selection, and (2) similar to the findings obtained in Experiment 2, the representational level at which object-based attentional select takes place can be influenced by top-down factors, such as the context in which selection takes place.

It is important to note, however, that, as predicted, the results obtained in Experiment 4 also suggest limits on the influence of top-down factors on attentional selection. The no-parsing point wrenches produced a same-object effect in both the N/N sequence and the $\mathrm{G} / \mathrm{N}$ sequence, suggesting that these stimuli were selected at the singleUC representational level regardless of whether they were preceded by selection at the single-UC or parsed representational level. However, perhaps this is not surprising since, due to the lack of well-defined concave discontinuities, it is unlikely that the no-parsing point wrenches would be represented at the parsed level. In any event, these results clearly suggest that the representational level at which object-based selection can take place is constrained by both the geometric and the surface characteristics of the stimuli and by top-down factors, such as context and expectancies.

\section{GENERAL DISCUSSION}

The results of the four experiments described here, when viewed in conjunction with other investigations of visual attention (Baylis \& Driver, 1993, 1995; Driver \& 
Baylis, 1995; Duncan, 1993; Kramer \& Jacobson, 1991; Logan, 1997; Tipper \& Behrmann, 1996), begin to define the representations from which object-based attentional selection can take place, as well as the influence of top-down and bottom-up factors on the selection process. In Experiment 1, the subjects identified two propertiesa bent end and an open end-that appeared either on a single wrench or on two briefly presented wrenches. A same-object effect was obtained for single-UC wrenches but not for multiple-UC wrenches, suggesting that attentional selection occurred at the single-UC representational level (see Figure 1). These results strongly suggest that $\mathrm{UC}$ regions can serve as representations for object-based attentional selection, and, therefore, along with the demonstrations provided by Palmer and Rock (1994a; see also Koffka, 1935), they provide support for the importance of uniform connectedness in perceptual organization and attention.

In Experiment 2, the subjects performed the same property identification task with the same multiple-UC wrenches employed in Experiment 1. However, in this situation, the subjects had to occasionally decide whether a secondary task probe-a single wrench--matched one of the two wrenches that had just been judged in terms of the two target properties. Under these conditions, a large and robust same-object effect was obtained for the multiple-UC wrenches, suggesting selection from the grouped representational level. Consistent with the results of previous experiments (Baylis \& Driver, 1993; Yantis, 1992), these data suggest that top-down factorsin the present case, the context in which the property identification task was performed - can influence the representational level from which object-based attentional selection takes place. However, unlike the results of previous experiments, the present findings suggest, as illustrated in Figure 1, that context can encourage a shift in the representational level from which attentional selection occurs. Thus, we suggest that, while the multipleUC stimuli were selected at the single-UC representational level in Experiment 1, as evidenced by the lack of a same-object effect, these same stimuli were selected at the grouped representational level in Experiment 2, as evidenced by the presence of a same-object effect.

In Experiment 3, we systematically manipulated the magnitude of the concave discontinuities that occurred at the point that the wrench ends were attached to the wrench shafts. As illustrated in Figure 4, the size of the sameobject effect varied inversely with the magnitude of the concave discontinuities, suggesting an increased likelihood of selection from the parsed representational level with increases in the salience of the object parts (D. D. Hoffman \& Singh, 1997). Thus, along with the work of Baylis and Driver (1995; Driver \& Baylis, 1995), these results suggest that object parts created by a preattentive segmentation of the visual array at points of concave discontinuities (Biederman, 1987; D. D. Hoffman \& Richards, 1984) can serve as representations that are available for attentional selection.
In Experiment 4, we capitalized on Ward's (1982) level readiness effect to examine whether the good-parsing point wrenches that failed to produce a same-object effect in Experiment 3 would reveal a same-object effect and therefore suggest selection from a single-UC representation rather than a parsed representation, when these stimuli were primed by selection of the no-parsing point wrenches. Indeed, this is what we found. These data suggest, like the findings obtained in Experiment 2 with the multiple-UC stimuli, that the context in which the identification task takes place can have an strong influence on the representational level from which object-based selection takes place. Thus, although the geometric and surface characteristics of the stimuli define the possible representations that can be derived from the visual array, it is the interaction between these image-based properties and top-down factors that determine the representations that will be selected by attention.

Where do all of these interesting effects leave us with regard to the original question that we posed-that is, what are the representations from which object-based attentional selection occurs? As illustrated in Figure 1, we believe that our data, and the data of others, argue for at least three levels of representation from which attention can select. Indeed, given the richness of the visual world, it seems quite likely that additional representations, defined perhaps by hierarchical grouping and parsing operations, are available for attentional selection. However, given our current state of knowledge, it would appear prudent to assume that three levels of representation are available for selection, at least for the present.

An interesting and important related question concerns the role of UC representations in perceptual organization and visual selective attention. Palmer and Rock (1994a, 1994b) argued that UC regions serve as the initial or entry level units into the part-whole organizational hierarchy, and that grouping and parsing operations are then performed on these primitive units. However, other researchers (Peterson, 1994) have argued that the research available at the present time is more consistent with the view that $\mathrm{UC}$ represents a principle of perceptual organization, albeit a powerful one, rather than the fundamental principle of organization. What do the data obtained in the present experiments have to say about this important issue? At the very least, we believe that our data suggest that UC is a powerful principle of perceptual organization and that it may be much more. First, the data obtained in Experiment 1 suggest that, even when a familiar object - a common everyday wrench with a handle that each of our subjects was able to rapidly recognize as such-is presented to subjects, the preferential level of selection is the single-UC representation. This was evidenced by the lack of a same-object effect for the multiple-UC stimuli in Experiment 1. Second, the level readiness priming effect obtained in Experiment 4 further suggests that selection at the single-UC representational level can have a strong influence on subsequent selection - in the present case of the good-parsing point 
wrench. Thus, both of these effects suggest that the presence of UC representations has a strong influence on object-based selective attention. However, on the other hand, even with briefly presented displays (i.e., $50 \mathrm{msec}$ in the present experiments) selection of UC regions is clearly not mandatory. Both geometric characteristics of stimuli, as evidenced by the good-parsing point wrenches in Experiment 3, and context, as evidenced by the multiple-UC wrenches in Experiment 2, can encourage attentional selection at other representational levels. Therefore, it would appear that at least three different types of representations within the part-whole hierarchy are available for attentional selection relatively soon after the presentation of visual displays.

There are at least two ways that future research might address whether UC regions serve as entry level units for attentional selection. First, the systematic manipulation of stimulus duration, starting with very brief presentations (e.g., $15 \mathrm{msec}$ ), along with the use of postdisplay masks, would seem to be one way to determine whether UC regions become available for attentional selection prior to grouped or parsed representations. Second, an examination of the magnitude and time course of priming effects, within the context of the level readiness paradigm, would appear to be another reasonable way to determine the relative strength of different levels of representation with regard to attentional selection. In any event, it should be clear that additional research will be required to determine whether uniform connectedness represents the fundamental principle of organization or, instead, another, albeit powerful, grouping principle.

In our theoretical sketch illustrated in Figure 1, we imply that attention can address only a single representational level at a time. This seems like a reasonable working hypothesis given previous demonstrations that attention can be preferentially directed to a single level in the global-local hierarchy (J. E. Hoffman, 1980; Kinchla et al., 1983; Ward, 1982), as well as evidence that attention can only be directed to a single location in the visual field at a particular point in time (C. W. Eriksen \& St. James, 1986; Posner, Snyder, \& Davidson, 1980; but see Kramer \& Hahn, 1995). This hypothesis is also consistent, in part, with the presence or absence of the same-object effect for the multiple-UC wrenches in Experiments 1 and 2 and the good-parsing point wrenches in Experiments 3 and 4 , as a function of the context in which the property identification task was performed. However, it is also conceivable that the variations in the magnitude of the same-object effect (see, for example, Figure 4), from which we have inferred the level of representation from which selection occurred, are consistent with parallel selection from multiple representational levels, perhaps with bias weights set on the basis of bottom-up and topdown factors. One way to address this issue in future research is to fit multinominal mixture distribution models to RT distributions obtained in different experimental conditions (Yantis, Meyer, \& Smith, 1991). Such a procedure should enable the determination of whether changes in the magnitude of the same-object effect are the result of changes in the mixture of the proportion of trials on which selection occurs from one or another of the representational levels or, instead, whether selection can occur simultaneously from multiple representational levels.

A related issue concerns the manner in which top-down and bottom-up factors interact to determine the representational level from which object-based attentional selection occurs (or if selection can occur from multiple representational levels concurrently, which representation wins the race to influence behavior). In Experiment 2, the finding of a same-object effect for the multiple-UC wrenches suggests that top-down influence in the form of context can influence the representation level from which selection occurs. However, the magnitude of the sameobject effect was smaller for the multiple-UC wrenches in Experiment 2 than it was for the single-UC wrenches in Experiment 1. A similar pattern of results was obtained in Experiment 4 for the good-parsing point wrenches. That is, the same-object effect for the good-parsing point wrenches in Experiment 4 was smaller than the sameobject effect for the no-parsing point wrenches in Experiments 3 and 4 . Thus, while the results of the present experiments clearly indicate that top-down factors can influence the representational level from which objectbased selection occurs, the magnitude of the same-object effect was smaller in the experiments in which top-down factors were introduced (i.e., Experiments 2 and 4) than when objects were defined by surface (Experiment 1 ) and geometric (Experiment 4) characteristics. ${ }^{6}$ Whether the difference in the magnitude of the same-object effect is attributable to the relative effectiveness of the top-down and bottom-up cues or to a general bias in bottom-up characteristics of objects is an important question for future research.

Another interesting issue with regard to object-based attentional selection is the role of space in selection. Clearly, our results suggest that selection was not occurring from an attentional window, spotlight, or zoom lens (Downing \& Pinker, 1985; C. W. Eriksen \& Yeh, 1985; Posner, 1980) that maintained a fixed shape regardless of the structure available in the visual field. That is, our finding of same-object effects, given the equal separation between target properties within and between objects, cannot be explained by a classical space-based attention model. There are, however, other ways in which space can play a role in attentional selection. For example, Vecera and Farah (1994; see also Vecera, 1994) suggested an important distinction between grouped-array selection and spatially invariant object-based selection. In grouped-array selection, attention may be directed within a spatiotopic representation defined by the contours of objects. Thus, within this framework, the shape of spatial attention is constrained only by the shape of the target objects rather than being confined to circular or elliptical distributions (see also Kramer et al., 1997, LaBerge, 1995, and Logan, 1997, for similar views of the 
role of space in object-based attentional selection). Another option is that spatial location plays no role in the selection process since attention selects objects from an internal representation in which they are coded in a spatially invariant fashion (Vecera \& Farah, 1994). At first glance, our failure to find spatial priming effects (i.e., faster or more accurate identification when the target properties appeared in the same location than when they appeared in different locations on two temporally contiguous trials) may be taken as evidence in favor of the spatially invariant object-based proposal. However, clearly there was sufficient time between subsequent trials in our tasks such that, if attention had been allocated spatially, it may have been reallocated, perhaps to encompass all possible target locations, by the time the next display appeared. Therefore, further investigation of this important issue will require a more systematic (and likely more finegrained) manipulation of the time between subsequent trials or the use of post-display probes (Cave \& Pashler, 1995; Kim \& Cave, 1995) or ERPs (Weber et al., 1997).

In summary, although there are clearly many unresolved issues concerning the processes that underlie and representations from which object-based selection occurs, the results obtained in the present experiments provide an important beginning in the explication of the influence of bottom-up and top-down factors in defining the manner in which objects are selectively attended in vision. Along with the results of others (Baylis \& Driver, 1995; Driver \& Baylis, 1995; Vecera \& Farah, 1994), the present findings suggest that attentional researchers no longer need to define objects available for selection in a post hoc manner but, instead, can begin to specify a priori the geometric and surface characteristics (Biederman, 1987; D. D. Hoffman \& Singh, 1997; Palmer \& Rock, 1994b) that define candidate objects and the influence top-down processes (Baylis \& Driver, 1993; Yantis, 1992), such as context and expectancies on the level of representations from which attentional selection will take place.

\section{REFERENCES}

Bashinski, H. S., \& Bacharach, V. R. (1980). Enhancement of perceptual sensitivity as the result of selectively attending to spatial locations. Perception \& Psychophysics, 28, 241-248.

BAYLIS, G. C. (1994). Visual attention and objects: Two-object cost with equal convexity. Journal of Experimental Psychology: Human Perception \& Performance, 20, 208-212.

Baylis, G. C., \& Driver, J. (1992). Visual parsing and response competition: The effect of grouping factors. Perception \& Psychophysics, 51, 145-162.

BAYLIS, G. C., \& DRIVER, J. (1993). Visual attention and objects: Evidence for hierarchical coding of location. Journal of Experimental Psychology: Human Perception \& Performance, 19, 451-470.

BAYLIS, G. C., \& Driver, J. (1995). One-sided edge assignment in vision: I. Figure-ground segregation and attention to objects. Current Directions in Psychological Science, 4, 140-146.

Behrmann, M., \& Moscovitch, M. (1994). Object-centered neglect in patients with unilateral neglect: Effects of left-right coordinates of objects. Journal of Cognitive Neuroscience, 6, 1-16.

BehrmanN, M., Zemel, R., \& Mozer, M. C. (1998). Object-based at- tention and occlusion: Evidence from normal subjects and a computational model. Journal of Experimental Psychology: Human Perception \& Performance, 24, 1011-1036.

BIEDERMAN, I. (1987). Recognition by components: A theory of object recognition. Psychological Review, 94, 115-147.

Cave, K. R., \& Pashler, H. (1995). Visual selection mediated by location: Selecting successive visual objects. Perception \& Psychophysics, 57, 421-432.

DAVIS, G., \& DRIVER, J. (1997). Spreading of visual attention to modally versus amodally completed regions. Psychological Science, 8, 275-281.

Downing, C. J., \& Pinker, S. (1985). The spatial structure of visual attention. In M. I. Posner \& O. S. M. Martin (Eds.), Attention and performance $X I$ (pp. 171-187). Hillsdale, NJ: Erlbaum.

DRIVER, J., \& BAYLIS, G. C. (1995). One-sided edge assignment in vision: 11. Part decomposition, shape description, and attention to objects. Current Directions in Psychological Science, 4, 201-206.

Driver, J., \& Halligan, P. (1991). Can visual neglect operate in object-centered coordinates? Cognitive Neuropsychology, 8, 475-496.

DUNCAN, J. (1984). Selective attention and the organization of visual information. Journal of Experimental Psychology: General, 113, 501517.

DUNCAN, J. (1993). Similarity between concurrent visual discriminations: Dimensions and objects. Perception \& Psychophysics, 54, 425-430.

EgLy, R., Driver, J., \& Rafal, R. (1994). Shifting visual attention between objects and locations: Evidence from normal and parietal lesion subjects. Journal of Experimental Psychology: General, 123, 161-177.

Egly, R., Rafal, R., Driver, J., \& Starrveveld, Y. (1994). Covert orienting in the split brain reveals hemispheric specialization for objectbased attention. Psychological Science, 5, 380-383.

EriKsen, B. A., \& EriKsen, C. W. (1974). Effects of noise letters upon the identification of a target letter in a nonsearch task. Perception $\&$ Psychophysics, 16, 143-149.

ERIKSEN, C. W., \& ST. JAMES, J. D. (1986). Visual attention within and around the field of focal attention: A zoom lens model. Perception \& Psychophysics, 40, 225-240.

ERIKSEN, C. W., \& YeH, Y. Y. (1985). Allocation of attention in the visual field. Journal of Experimental Psychology: Human Perception \& Performance, 11, 583-597.

Hillyard, S. A., Anllo-Vento, L., Clark, V., Heinze, H., Luck, S. J., \& MAngun, G. R. (1996). Neuroimaging approaches to the study of visual attention. In A. F. Kramer, M. Coles, \& G. Logan (Eds.), Converging operations in the study of visual selective attention (pp. 107-138). Washington, DC: American Psychological Association.

Hoffman, D. D., \& Richards, W. (1984). Parts of recognition. Cognition, 18, 65-96.

Hoffman, D. D., \& Singh, M. (1997). Salience of visual parts. Cognition, 63, 29-78.

HoffMan, J. E. (1980). Interaction between global and local levels of form. Journal of Experimental Psychology: Human Perception \& Performance, 6, 222-234.

Hoffman, J. E., Houck, M. R., McMillian, F. W., Simons, R. F., \& OATMAN, L. C. (1985). Event related potentials elicited by automatic targets: A dual task analysis. Journal of Experimental Psychology: Human Perception \& Performance, 11, 50-61.

Hoffman, J. E., \& Nelson, B. (1981). Spatial selectivity in visual search. Perception \& Psychophysics, 30, 283-290.

Humphreys, G., Olson, A., Romani, C., \& Riddoch, M. (1996). Competitive mechanisms of selection by object and space: A neuropsychological approach. In A. F. Kramer, M. Coles, \& G. Logan (Eds.), Converging operations in the study of visual selective attention (pp. 365-393). Washington, DC: APA.

HumpHREYs, G., \& RidDoch, M. (1993). Interactions between object and space vision revealed through neuropsychology. In D. Meyer \& S. Kornblum (Eds.), Attention and performance XIV (pp. 143-162). Hillsdale, NJ: Erlbaum.

Johnson, D. N., \& YanTIS, S. (1995). Allocating visual attention: Tests of a two-process model. Journal of Experimental Psychology: Human Perception \& Performance, 21, 1376-1390. 
Kahneman, D., \& Henik, A. (1981). Perceptual organization and attention. In M. Kubovy \& J. R. Pomerantz (Eds.), Perceptual organization (pp. 181-211). Hillsdale, NJ: Erlbaum.

Kahneman, D., Treisman, A., \& Gibbs, B. (1992). The reviewing of object files: Object specific integration of information. Cognitive Psychology, 24, 175-219.

KIM, M. S., \& CAVE, K. (1995). Spatial attention in visual search for features and feature conjunctions. Psychological Science, 6, 376-380.

Kinchla, R. A., Solis-Macias, V., \& Hoffman, J. [E.] (1983). Attending to different levels of structure in the visual image. Perception \& Psychophysics, 33, 1-10.

KoffKa, K. (1935). Principles of Gestalt psychology. New York: Harcourt Brace.

KRAMER, A. F., \& HAHN, S. (1995). Splitting the beam: Distribution of attention over noncontiguous regions of the visual field. Psychological Science, 6, 381-386.

KRAmER, A. F., \& JACOBSON, A. (1991). Perceptual organization and focused attention: The role of objects and proximity in visual processing. Perception \& Psychophysics, 50, 267-284.

Kramer, A. F., \& WATSON, S. E. (1996). Object-based visual selection and the principle of uniform connectedness. In A. F. Kramer, M. Coles, \& G. Logan (Eds.), Converging operations in the study of visual selective attention (pp. 395-414). Washington, DC: American Psychological Association.

Kramer, A. F., Weber, T., \& Watson, S. E. (1997), Object-based attentional selection: Grouped-arrays or spatially-invariant representations? Journal of Experimental Psychology: General, 126, 3-13.

Kramer, A. F., Wickens, C. D., \& DonChIN, E. (1985). The processing of stimulus properties: Evidence for dual task integrality. Journal of Experimental Psychology: Human Perception \& Performance, 11, 393-408.

LABERGE, D. (1995). Computational and anatomical models of selective attention in object identification. In M. S. Gazzaniga (Ed.), The cognitive neurosciences (pp. 649-663). Cambridge, MA: MIT Press.

LABERGE, D., \& BROWN, V. (1989). Theory of attentional operation in shape identification. Psychological Review, 96, 101-124.

LAvie, N., \& Driver, J. (1996). On the spatial extent of attention in object-based visual selection. Perception \& Psychophysics, 58, 12381251.

LoGAN, G. (1997). The CODE theory of visual attention: An integration of space-based and object-based attention. Psychological Review, $103,603-649$.

LowE, D. (1985). Perceptual organization and visual recognition. Amsterdam: Kluwer.

MANGUn, G. R., \& HillyaRd, S. A. (1990). Electrophysiological studies of visual selective attention in humans. In A. Scheibel \& A. Wechsler (Eds.), Neurobiology of higher cognitive function (pp. 271-295). New York: Guilford

Marr, D. (1982). Vision. San Francisco: W. H. Freeman.

MARR, D., \& NishihaRA, H. (1978). Representation and recognition of the spatial organization of 3-dimensional shapes. Proceedings of the Royal Society of London: Series B, 200, 269-294.

Moore, C. M., \& EgETH, H. (1997). Perception without attention: Evidence of grouping under conditions of inattention. Journal of Experimental Psychology: Human Perception \& Performance, 23, 339-352.

NAvon, D. (1977). Forest before the trees: The precedence of global features in perception. Cognitive Psychology, 9, 353-383.

NeISSER, U. (1967). Cognitive psychology. Englewood Cliffs, NJ: Prentice-Hall.

PALMER, S. (1977). Hierarchical structure in perceptual representation. Cognitive Psychology, 9, 441-474.

PALMER, S., \& RoCK, I. (1994a). On the nature and order of organizational processing: A reply to Peterson. Psychonomic Bulletin \& Review, 1, 515-519.

PALmer, S., \& RoCK, I. (1994b). Rethinking perceptual organization: The role of uniform connectedness. Psychonomic Bulletin \& Review, 1, 29-55.

Palmer, S., Rosch, E., \& Chase, P. (1981). Canonical perspective and the perception of objects. In J. Long \& A. Baddeley (Eds.), Attention and performance IX (pp. 135-151). Hillsdale, NJ: Erlbaum.

Peterson, M. A. (1994). The proper placement of uniform connectedness. Psychonomic Bulletin \& Review, 1, 509-514.
POSNER, M. I. (1980). Orienting of attention. Quarterly Journal of Experimental Psychology, 32, 3-25.

Posner, M. I., SNyder, C. R. R., \& Davidson, B. J. (1980). Attention and the detection of signals. Journal of Experimental Psychology: General, 109, 160-174

Posner, M. I., Walker, J., Friedrich, F., \& Rafal, R. (1984). Effects of parietal lobe injury on covert orienting of visual attention. Journal of Neuroscience, 4, 1863-1874.

RiDDOCH, M., \& HuMPhREYS, G. (1983). The effect of cueing on unilateral neglect. Neuropsychologia, 21, 588-599.

ROBERTSON, L. C. (1996). Attentional persistence for features of hierarchical patterns. Journal of Experimental Psychology: Human Perception \& Performance, 125, 227-249.

Robertson, L. C., Egly, R., Lamb, M. R., \& Kerth, L. (1993). Spatial attention and cueing to global and local levels of hierarchical structure. Journal of Experimental Psychology: Human Perception \& Performance, 19, 471-487.

Rorden, C., Mattingley, J. B., Karnath, H. O., \& Driver, J. (1997). Visual extinction and prior entry: Impaired perception of temporal order with intact motion perception after unilateral parietal damage. Neuropsychologia, 35, 421-433.

RUTHRUFF, E. , \& MilLER, J. (1995). Negative priming depends on ease of selection. Perception \& Psychophysics, 57, 715-723.

SPERling, G., \& MELCher, M. (1978). Visual search, visual attention and the attention operating characteristic. In J. Requin (Eds.), Attention and performance VII. Hillsdale, NJ: Erlbaum.

StUarT, G. W., MarufF, P., \& CuRrie, J. (1997). Object-based attention in luminance increment detection? Neuropsychologia, 35, 843-853.

TIPPER, S. P., \& BEHRMANN, M. (1996). Object-centered not scenebased visual neglect. Journal of Experimental Psychology: Human Perception \& Performance, 22, 1261-1278.

Tipper, S. P., Brehaut, J. C., \& Driver, J. (1990). Selection of moving and static objects for the control of spatially directed attention Journal of Experimental Psychology: Human Perception \& Performance, 16, 492-504

TtPPER, S. P., Driver, J,, \& WeAVER, B. (1991). Object-centered inhibition of return of visual attention. Quarterly Journal of Experimental Psychology, 43, 289-298.

Treisman, A., Kahneman, D., \& Burkell, J. (1983). Perceptual objects and the cost of filtering. Perception \& Psychophysics, 33, 527-532.

VECERA, S. P. (1994). Grouped locations and object-based attention: Comment on Egly, Driver and Rafal (1994). Journal of Experimental Psychology: General, 123, 316-320.

VeCERA, S. P., \& FARAH, M. J. (1994). Does visual attention select objects or locations? Journal of Experimental Psychology: General, 123, 146-160.

WARD, L. (1982). Determinants of attention to local and global features of visual forms. Journal of Experimental Psychology: Human Perception \& Performance, 8, 562-581.

WeAVER, B., LUPIÁÑEz, J., \& WATSON, F. L. (1998). The effects of practice on object-based, location-based, and static-display inhibition of return. Perception \& Psychophysics, 60, 993-1003.

Weber, T., Kramer, A. F., \& Miller, G. (1997). Selective processing of superimposed objects: An electrophysiological analysis of objectbased attentional selection. Biological Psychology, 45, 159-182.

WERTHEIMER, M. (1923). Untersuchungen zur Lehre von der Gestalt [Examinations of Gestalt teachings]. Psychologische Forschung, 4 301-350.

YANTIS, S. (1992). Multielement visual tracking: Attention and perceptual organization. Cognitive Psychology, 24, 295-340.

Yantis S., Meyer, D. E., \& Smith, J. E. K. (1991). Analysis of multinominal mixture distributions: New tests for stochastic models of cognition and action. Psychological Bulletin, 110, 350-374.

YANTIS, S., \& MOORE, C. (1995, November). Spreading of visual attention behind an occluding surface. Paper presented at the annual meeting of the Psychonomic Society, Los Angeles.

\section{NOTES}

1. One may wonder why we ran so many subjects in our experiments. Was it due to large intersubject variability and, therefore, low power to detect significant differences among experimental conditions? The an- 
swer is an unequivocal no. The main results that we report in this paper were significant with 16 or fewer subjects. Fairly large numbers of subjects were run in the reported experiments in an effort to enable us to also examine the relationship between individual differences in objectbased attentional selection and other perceptual and cognitive processes. These data will be reported elsewhere.

2. All post hoc comparisons were performed with the Bonferroni $t$ test and were significant at $p<.05$.

3. We also failed to find any evidence of spatial priming (i.e., faster and/or more accurate performance when the locations of the target properties were the same across trials than when the locations of the target properties varied across trials) in the other three experiments reported in the present paper.

4. An interesting question is whether the representation from which selection takes place in Experiment 2 involves unitary perceptual objects, as would be suggested by the example presented in the grouped representation in Figure 1, or whether it entails a part-based representation in which the parts are represented along with their relationships (i.e., the open end is to the left of the blue-stripped handle, which, in turn, is to the left of the bent end). The present data do not enable us to discriminate between these two possibilities, and, therefore, additional research will be required to address this issue. However, in either case (i.e., unitary perceptual object or part-based representation with tagged relationships), the representation available for selection at the grouped representation level differs from that available at the single-UC representation level.

5. Another possibility is that parsing at concave discontinuities is mandatory (i.e., at least when concave discontinuities are above some minimum perceptible threshold), but representations are still created and are available for object-based attentional selection at other levels of the representational hierarchy. In this case, the level readiness effect would bias selection at the single-UC representational level when the no-parsing point wrench precedes the good-parsing point wrench.

6. One unanswered question is whether the context effects obtained in Experiments 2 and 4 can be explained by a system that learns which representations are the most efficient for a particular task without requiring any top-down input. Such an explanation would appear to require an increase in the magnitude of the same-object effect with practice. However, we failed to find any evidence for such an effect in Experiments 2 and 4 . That is, the magnitude of the same-object effect did not change with practice for the multiple-UC wrenches in Experiment 2 or for the good-parsing point wrenches (when preceded by the poor-parsing point wrenches) in Experiment 4.

(Manuscript received August 22, 1997; revision accepted for publication December 24,1997 .) 